\title{
Comparative analysis on bifurcation of four-neuron fractional ring networks without or with leakage delays
}

\author{
Zhouhong Li ${ }^{1 *}$ (D), Chengdai Huang ${ }^{2}$ and Yuan Zhang ${ }^{1}$
}

"Correspondence: Izh@yxnu.edu.cn 'Department of Mathematics, Yuxi Normal University, Yuxi, China Full list of author information is available at the end of the article

\begin{abstract}
This paper is concerned with the problem of bifurcation for a ring fractional Hopfield neural network with leakage time delay and communication time delay. The stability and the Hopf bifurcations of such a network without and with time delays are investigated by analyzing the associated characteristic equations. Specifically, some criteria for the occurrence of Hopf bifurcations at the trivial steady state are established. It is shown that the dynamical property of the network is not only crucially dependent on the communication time delay, but also significantly influenced by the leakage time delay. Furthermore, the effects of the order on the Hopf bifurcation are numerically demonstrated. Finally, four numerical examples are provided to illustrate the feasibility of the theoretical results.
\end{abstract}

Keywords: Leakage delay; Stability; Hopf bifurcation; Fractional order; A ring of neural network

\section{Introduction}

The studies for various Hopfield neural networks (HNNs) have been continuously active over the past three decades because of their successful applications in numerous areas, for instance, optimizations, signal processing, image processing, solving nonlinear algebraic equations, pattern recognitions, associative memories [1-5]. Since the applications of HNNs rely heavily on network dynamics, many efforts have been undertaken to investigate their dynamical properties and a lot of valuable results have been reported, including stability [6], oscillation [7], bifurcation [8-10], chaos [11], and synchronization [12, 13] and the references.

One major and often encountered difficulty in the analysis of neural network dynamics is the ubiquity of time delays that can result in instability, oscillation, periodic solution, anti-periodic solution, almost periodic solution, quasi-periodic solution, and even give rise to multistability and chaotic motion. Among them, the time delays resulting from the communication and response of neurons are regarded as a critical player due to the finite switching speed of amplifiers and the non-instantaneous signal transmission between neurons [14]. Over the years, the study of dynamics of HNNs or population with such time delays has received considerable interest of many researchers $[2-5,7,11,15]$. Additionally, it has been observed that a typical time delay called leakage delay also has important

(c) The Author(s) 2019. This article is distributed under the terms of the Creative Commons Attribution 4.0 International License (http://creativecommons.org/licenses/by/4.0/), which permits unrestricted use, distribution, and reproduction in any medium, provided you give appropriate credit to the original author(s) and the source, provide a link to the Creative Commons license, and indicate if changes were made. 
consequences on dynamics of neural networks [16-21]. In particular, the leakage delay in a negative feedback terms can drive a stable system to be unstable [22]. It is therefore also of great significance to clarify the dynamics of HNNs subject to leakage delays.

In 2009, $\mathrm{Hu}$ and Huang [23] investigated a ring of HNN with four neurons and delays, which is described as follows:

$$
\left\{\begin{array}{l}
\dot{x_{1}}(t)=-r_{1} x_{1}(t)+g_{1}\left(x_{1}(t)\right)+f_{1}\left(x_{4}\left(t-\tau_{2}\right)\right)+f_{1}\left(x_{2}\left(t-\tau_{2}\right)\right), \\
\dot{x_{2}}(t)=-r_{2} x_{2}(t)+g_{2}\left(x_{2}(t)\right)+f_{2}\left(x_{1}\left(t-\tau_{1}\right)\right)+f_{2}\left(x_{3}\left(t-\tau_{1}\right)\right), \\
\dot{x_{3}}(t)=-r_{3} x_{3}(t)+g_{3}\left(x_{3}(t)\right)+f_{3}\left(x_{2}\left(t-\tau_{2}\right)\right)+f_{3}\left(x_{4}\left(t-\tau_{2}\right)\right), \\
\dot{x_{4}}(t)=-r_{4} x_{4}(t)+g_{4}\left(x_{4}(t)\right)+f_{4}\left(x_{3}\left(t-\tau_{1}\right)\right)+f_{4}\left(x_{1}\left(t-\tau_{1}\right)\right),
\end{array}\right.
$$

where $\dot{x}=\mathrm{d} x / \mathrm{d} t ; x_{i}(t)$ represents the state of the $i$ th neuron at time $t ; r_{i} \geq 0$ is the internal decay rate; $f_{i}$ is the connection function between neurons; $g_{i}$ represents the nonlinear feedback function; $\tau_{j} \geq 0$ is the communication time delay; and $i=1,2,3,4, j=1,2$. By using the associated characteristic equation, the stability and Hopf bifurcations of the HNN are studied, as well as the stability and direction on the Hopf bifurcation are determined by employing the normal form method and the center manifold reduction. For more ring networks research results, one can read the references $[8,24-26]$ and the references cited therein.

Fractional calculus, a classical mathematical notion that has a history of over 300 years, is a generalization of the ordinary differentiation and integration to arbitrary non-integer order, having been demonstrated to play important roles in physics, biology and engineering [27-35]. In fact, the importance of fractional calculus is reflected in three main points: first, the orders of derivatives and integrals in fractional calculus are real numbers; second, fractional-order derivative acts as an effective measure for the description of memory and hereditary properties of various materials and processes; and third, the fractional-order derivative makes a real object models more accurately than the integer order. Based on these advantages, fractional calculus has been proposed to model, design, and control various neural networks in recent years. For instance, several works concerning fractional neural networks have appeared recently: undamped oscillations generated by Hopf bifurcations in fractional-order recurrent neural networks with Caputo derivative were studied in [36, 37]; for a fractional BAM neural network with leakage delay, conditions for the Hopf bifurcation were discussed in [38], and so on.

Oscillations are ubiquitous in dynamic neuronal networks and play critical roles in fundamental processes such as controlling dynamics of neurons at subthreshold potentials, regulating rhythmic neuronal ensembles within local networks, and determining global oscillations measured by electroencephalography [12]. It is well known that Hopf bifurcations, which include supercritical and subcritical Hopf bifurcations, can help us to efficiently design biochemical oscillators. In this regard, it is important to note that most of the results on Hopf bifurcation theory of integer-order neural networks cannot be simply generalized to those for the cases of fractional neural networks because of the substantial differences between integer-order system and fractional-order system. To the best of our knowledge, up to today only a few results on the Hopf bifurcation of fractional-order system have been reported, and thus, it is still an open problem to study Hopf bifurcations of fractional-order dynamical systems [39]. This finding motivates the search for the 
properties of bifurcated oscillations of a ring of fractional-order neural network with four neurons further.

Based on the above motivations, the present work is devoted to the study of stability and Hopf bifurcation for a delayed ring of fractional-order neural network with four neurons and leakage delays. The main contributions can be summarized in three aspects:

(1) A new delayed four-neuron fractional-order ring network with leakage delays is proposed.

(2) Two important dynamical properties-stability and oscillation—of the four neurons fractional-order ring networks without and with explicit leakage delays are investigated.

(3) The effects of the order on the Hopf bifurcation are discussed.

The rest of this paper is organized as follows. In Sect. 2, several definitions and lemma of fractional-order calculus are recalled. In Sect. 3, the discussed models are proposed. In Sect. 4, by analyzing the associated characteristic equation, the local stability of the trivial steady state for the delayed fractional-order HNN is examined. Moreover, the existence of the Hopf bifurcation of the delayed fractional-order HNN without and with leakage time delays is established. In Sect. 5, illustrative examples are provided to demonstrate the theoretical results. Some conclusions are given in the last section.

\section{Preliminaries}

In this section, we introduce some definitions and lemmas of fractional derivatives, which serve as a basis for the proofs of the main result of Sect. 4 .

Generally, there exist mainly three widely used fractional operators, namely the Grünwald-Letnikov definition, the Riemann-Liouville definition, and the Caputo definition. Since the Caputo derivative only requires the initial conditions, which are based on integer-order derivative and represents well-understood features of physical situation, it is more applicable to real world problems. With this notion in mind, we shall use the Caputo fractional-order derivative to model and analyze the stability of the proposed fractionalorder algorithms in this paper.

Definition 2.1 ([28]) The fractional integral of order $\phi$ for a function $g(t)$ is defined as follows:

$$
I^{\phi} g(t)=\frac{1}{\Gamma(\phi)} \int_{t_{0}}^{t}(t-s)^{\phi-1} g(s) d s
$$

here, $t \geq t_{0}, \phi>0$, and $\Gamma(\cdot)$ is the gamma function satisfying $\Gamma(s)=\int_{0}^{\infty} t^{s-1} e^{-t} d \mathrm{t}$.

Definition 2.2 ([28]) Caputo fractional derivative of order $\phi$ for a function $g(t) \in C^{n}\left(\left[t_{0}\right.\right.$, $\infty), R$ ) is defined in the following form:

$$
D^{\phi} g(t)=\frac{1}{\Gamma(n-\phi)} \int_{t_{0}}^{t} \frac{g^{(n)}(s)}{(t-s)^{\phi-n+1}} d \mathrm{~s}
$$

here, $t \geq t_{0}$, and $n-1 \leq \phi<n, n \in N^{+}$. 
Moreover, if $0<\phi<1$, then

$$
D^{\phi} g(t)=\frac{1}{\Gamma(1-\phi)} \int_{t_{0}}^{t} \frac{g^{\prime}(s)}{(t-s)^{\phi}} d \mathrm{~s}
$$

Lemma 2.1 ([29]) For the following autonomous system

$$
D^{\phi} y=J y, \quad y(0)=y_{0}
$$

in which $0<\phi<1, y \in R^{n}, A \in R^{n \times n}$ is asymptotically stable if and only if $\left|\arg \left(\lambda_{i}\right)\right|>\phi \pi / 2$ $(i=1,2, \ldots, n)$, then each component of the states decays towards 0 like $t^{-\phi}$. Furthermore, this system is stable if and only if $\left|\arg \left(\lambda_{i}\right)\right| \geq \phi \pi / 2$ and those critical eigenvalues that satisfy $\left|\arg \left(\lambda_{i}\right)\right|=\phi \pi / 2$ have geometric multiplicity one.

\section{Model description}

This paper considers the following ring fractional HNN with four neurons and time delays in leakage terms:

$$
\left\{\begin{array}{l}
D^{\phi_{1}} x_{1}(t)=-r_{1} x_{1}(t-\sigma)+a g_{1}\left(x_{1}(t)\right)+b_{1} f_{1}\left(x_{4}\left(t-\tau_{2}\right)\right)+c_{1} f_{1}\left(x_{2}\left(t-\tau_{2}\right)\right), \\
D^{\phi_{2}} x_{2}(t)=-r_{2} x_{2}(t-\sigma)+a g_{2}\left(x_{2}(t)\right)+b_{2} f_{2}\left(x_{1}\left(t-\tau_{1}\right)\right)+c_{2} f_{2}\left(x_{3}\left(t-\tau_{1}\right)\right), \\
D^{\phi_{3}} x_{3}(t)=-r_{3} x_{3}(t-\sigma)+a g_{3}\left(x_{3}(t)\right)+b_{3} f_{3}\left(x_{2}\left(t-\tau_{2}\right)\right)+c_{3} f_{3}\left(x_{4}\left(t-\tau_{2}\right)\right), \\
D^{\phi_{4}} x_{4}(t)=-r_{4} x_{4}(t-\sigma)+a g_{4}\left(x_{4}(t)\right)+b_{4} f_{4}\left(x_{3}\left(t-\tau_{1}\right)\right)+c_{4} f_{4}\left(x_{1}\left(t-\tau_{1}\right)\right),
\end{array}\right.
$$

where $\phi_{i} \in(0,1](i=1,2,3,4)$ is fractional order; $x_{i}(t)(i=1,2,3,4)$ represents state variables; $r_{i} \geq 0(i=1,2,3,4)$ specifies the internal decay rate; $a, b_{i}, c_{i}(i=1,2,3,4)$ denote the connection weights; $f_{i}(\cdot)$ is the connection function between neurons; $g_{i}(\cdot)(i=1,2,3,4)$ represents the nonlinear feedback function; $\sigma$ is the leakage delay; $\tau_{1}$ and $\tau_{2}$ are the communication time delays.

Remark 3.1 In fact, if $\phi_{i}=1(i=1,2,3,4)$, the fractional-order system (3.1) changes into the following integer-order system:

$$
\left\{\begin{array}{l}
\dot{x_{1}}(t)=-r_{1} x_{1}(t-\sigma)+a g_{1}\left(x_{1}(t)\right)+b_{1} f_{1}\left(x_{4}\left(t-\tau_{2}\right)\right)+c_{1} f_{1}\left(x_{2}\left(t-\tau_{2}\right)\right), \\
\dot{x_{2}}(t)=-r_{2} x_{2}(t-\sigma)+a g_{2}\left(x_{2}(t)\right)+b_{2} f_{2}\left(x_{1}\left(t-\tau_{1}\right)\right)+c_{2} f_{2}\left(x_{3}\left(t-\tau_{1}\right)\right), \\
\dot{x_{3}}(t)=-r_{3} x_{3}(t-\sigma)+a g_{3}\left(x_{3}(t)\right)+b_{3} f_{3}\left(x_{2}\left(t-\tau_{2}\right)\right)+c_{3} f_{3}\left(x_{4}\left(t-\tau_{2}\right)\right), \\
\dot{x_{4}}(t)=-r_{4} x_{4}(t-\sigma)+a g_{4}\left(x_{4}(t)\right)+b_{4} f_{4}\left(x_{3}\left(t-\tau_{1}\right)\right)+c_{4} f_{4}\left(x_{1}\left(t-\tau_{1}\right)\right) .
\end{array}\right.
$$

In this work, for the sake of simplicity, we discuss the fractional-order system (3.1) when $\sigma=\tau_{1}=\tau_{2}=\tau, \phi=\phi_{1}=\phi_{2}=\phi_{3}=\phi_{4}$, and so system (3.1) can be rewritten as

$$
\left\{\begin{array}{l}
D^{\phi} x_{1}(t)=-r_{1} x_{1}(t-\tau)+a g_{1}\left(x_{1}(t)\right)+b_{1} f_{1}\left(x_{4}(t-\tau)\right)+c_{1} f_{1}\left(x_{2}(t-\tau)\right), \\
D^{\phi} x_{2}(t)=-r_{2} x_{2}(t-\tau)+a g_{2}\left(x_{2}(t)\right)+b_{2} f_{2}\left(x_{1}(t-\tau)\right)+c_{2} f_{2}\left(x_{3}(t-\tau)\right), \\
D^{\phi} x_{3}(t)=-r_{3} x_{3}(t-\tau)+a g_{3}\left(x_{3}(t)\right)+b_{3} f_{3}\left(x_{2}(t-\tau)\right)+c_{3} f_{3}\left(x_{4}(t-\tau)\right), \\
D^{\phi} x_{4}(t)=-r_{4} x_{4}(t-\tau)+a g_{4}\left(x_{4}(t)\right)+b_{4} f_{4}\left(x_{3}(t-\tau)\right)+c_{4} f_{4}\left(x_{1}(t-\tau)\right) .
\end{array}\right.
$$


Moreover, when system (3.2) does not involve leakage time delay, then system (3.2) can be described by

$$
\left\{\begin{array}{l}
D^{\phi} x_{1}(t)=-r_{1} x_{1}(t)+a g_{1}\left(x_{1}(t)\right)+b_{1} f_{1}\left(x_{4}(t-\tau)\right)+c_{1} f_{1}\left(x_{2}(t-\tau)\right), \\
D^{\phi} x_{2}(t)=-r_{2} x_{2}(t)+a g_{2}\left(x_{2}(t)\right)+b_{2} f_{2}\left(x_{1}(t-\tau)\right)+c_{2} f_{2}\left(x_{3}(t-\tau)\right), \\
D^{\phi} x_{3}(t)=-r_{3} x_{3}(t)+a g_{3}\left(x_{3}(t)\right)+b_{3} f_{3}\left(x_{2}(t-\tau)\right)+c_{3} f_{3}\left(x_{4}(t-\tau)\right), \\
D^{\phi} x_{4}(t)=-r_{4} x_{4}(t)+a g_{4}\left(x_{4}(t)\right)+b_{4} f_{4}\left(x_{3}(t-\tau)\right)+c_{4} f_{4}\left(x_{1}(t-\tau)\right) .
\end{array}\right.
$$

Accordingly, the primary objective of this paper is to study the stability and the Hopf bifurcations of networks (3.2) and (3.3) by taking time delay as the bifurcation parameter through the approach of stability analysis [27]. Moreover, the effects of the order on the creation of bifurcation for the two proposed models are also numerically discussed.

Throughout of this paper, some basic assumptions are presented first.

(C1) $f_{i}, g_{i} \in \mathrm{C}(R, R), f_{i}(0)=g_{i}(0)=0, x f_{i}(x)>0, x g_{i}(x)>0(i=1,2,3,4)$ for $x \neq 0$.

\section{Stability and bifurcation analysis}

\subsection{Existence of bifurcation without leakage delays}

In this subsection, by applying the previous analytic technique, we shall investigate the stability and bifurcation of system (3.3) by taking communication time delay as the bifurcation parameter. Accordingly, it is easy to show that the origin is an equilibrium point of system (3.3) under assumption (C1). The linearization of (3.3) at the origin is given by

$$
\left\{\begin{array}{l}
D^{\phi} x_{1}(t)=-r_{1} x_{1}(t)+a x_{1}(t)+m_{1} x_{4}(t-\tau)+n_{1} x_{2}(t-\tau) \\
D^{\phi} x_{2}(t)=-r_{2} x_{2}(t)+a x_{2}(t)+m_{2} x_{1}(t-\tau)+n_{2} x_{3}(t-\tau) \\
D^{\phi} x_{3}(t)=-r_{3} x_{3}(t)+a x_{3}(t)+m_{3} x_{2}(t-\tau)+n_{3} x_{4}(t-\tau) \\
D^{\phi} x_{4}(t)=-r_{4} x_{4}(t)+a x_{4}(t)+m_{4} x_{3}(t-\tau)+n_{4} x_{1}(t-\tau)
\end{array}\right.
$$

whose characteristic equation is

$$
\operatorname{det}\left(\begin{array}{cccc}
s^{\phi}+k_{1} & -n_{1} e^{s \tau} & 0 & -m_{1} e^{-s \tau} \\
-m_{2} e^{-s \tau} & s^{\phi}+k_{2} & n_{2} e^{-s \tau} & 0 \\
0 & m_{3} e^{-s \tau} & s^{\phi}+k_{3} & -n_{3} e^{-s \tau} \\
-n_{4} e^{-s \tau} & 0 & -m_{4} e^{-s \tau} & s^{\phi}+k_{4}
\end{array}\right)=0
$$

where $k_{i}=r_{i}-a g_{i}^{\prime}(0), m_{i}=b_{i} f_{i}^{\prime}(0), n_{i}=c_{i} f_{i}^{\prime}(0)(i=1,2,3,4)$.

By (4.2), we have

$$
P_{1}(s)+P_{2}(s) e^{-2 s \tau}+P_{3}(s) e^{-4 s \tau}=0,
$$

where

$$
\begin{aligned}
P_{1}(s)= & s^{4 \phi}+\left(k_{1}+k_{2}+k_{3}+k_{4}\right) s^{3 \phi}+\left(k_{1} k_{2}+k_{1} k_{3}+k_{1} k_{4}+k_{2} k_{3}+k_{2} k_{4}+k_{3} k_{4}\right) s^{2 \phi} \\
& +\left(k_{1} k_{2} k_{3}+k_{1} k_{2} k_{4}+k_{1} k_{3} k_{4}+k_{2} k_{3} k_{4}\right) s^{\phi}+k_{1} k_{2} k_{3} k_{4},
\end{aligned}
$$




$$
\begin{aligned}
P_{2}(s)= & -\left(m_{1} n_{4}+m_{2} n_{1}+m_{3} n_{2}\right) s^{2 \phi}+\left(m_{3} n_{2} k_{4}+m_{4} n_{3} k_{2}+m_{1} n_{4} k_{2}+m_{4} n_{3} k_{1}\right. \\
& \left.+m_{2} n_{1} k_{4}+m_{1} n_{4} k_{3}+m_{2} n_{1} k_{3}+m_{3} n_{2} k_{1}\right) s^{\phi}-\left(m_{4} n_{3} k_{1} k_{2}+m_{2} n_{1} k_{3} k_{4}\right. \\
& \left.+m_{1} n_{4} k_{2} k_{3}+m_{3} n_{2} k_{1} k_{4}\right), \\
P_{3}(s)= & m_{1} m_{3} n_{2} n_{4}+m_{2} m_{4} n_{1} n_{3}-m_{1} m_{2} m_{3} m_{4}-n_{1} n_{2} n_{3} n_{4} .
\end{aligned}
$$

Let $P_{1}(s)=A_{1}+i B_{1}, P_{2}(s)=A_{2}+i B_{2}, P_{3}(s)=A_{3}$, and from Eq. (4.3), we have

$$
\left(A_{1}+i B_{1}\right) e^{2 s \tau}+\left(A_{2}+i B_{2}\right)+A_{3} e^{-2 s \tau}=0
$$

Let $s=i w=w\left(\cos \frac{\pi}{2}+i \sin \frac{\pi}{2}\right)(\omega>0)$ be a root of Eq. (4.4). Substituting $s$ into Eq. (4.4) and separating the real and imaginary parts yields the following equations:

$$
\left\{\begin{array}{l}
\left(A_{1}+A_{3}\right) \cos 2 w \tau+B_{1} \sin 2 w \tau=-A_{2} \\
B_{1} \cos 2 w \tau+\left(A_{1}-A_{3}\right) \sin 2 w \tau=-B_{2}
\end{array}\right.
$$

which lead to

$$
\left\{\begin{array}{l}
\cos 2 w \tau=-\frac{A_{2}\left(A_{1}-A_{3}\right)+B_{1} B_{2}}{A_{1}^{2}+B_{1}^{2}-A_{3}^{2}}=\mathcal{F}(w), \\
\sin 2 w \tau=-\frac{B_{2}\left(A_{1}+A_{3}\right)-A_{2} B_{1}}{A_{1}^{2}+B_{1}^{2}-A_{3}^{2}}=\mathcal{G}(w) .
\end{array}\right.
$$

It is easy to see that

$$
\mathcal{F}^{2}(w)+\mathcal{G}^{2}(w)=1
$$

From (4.6), one can obtain

$$
\tau^{(l)}=\frac{1}{2 w}[\arccos \mathcal{F}(w)+2 l \pi], \quad l=0,1,2, \ldots
$$

Define the bifurcation point

$$
\tau_{0}^{*}=\min \left\{\tau^{(l)}\right\}, \quad l=0,1,2, \ldots
$$

To theoretically gain the sufficient conditions for the Hopf bifurcation, we assume that the following assumptions hold:

(C2) Eq. (4.7) has no positive real root;

(C3) Eq. (4.7) has at least a positive real root.

Denote

$$
\Xi_{1}=\Pi_{1}, \quad \Xi_{2}=\left|\begin{array}{cc}
\Pi_{1} & 1 \\
\Pi_{3} & \Pi_{2}
\end{array}\right|, \quad \Xi_{3}=\left|\begin{array}{ccc}
\Pi_{1} & 1 & 0 \\
\Pi_{3} & \Pi_{2} & \Pi_{1} \\
0 & \Pi_{4} & \Pi_{3}
\end{array}\right|, \quad \Xi_{4}=\Pi_{4} \Delta_{3},
$$

where

$$
\Pi_{1}=k_{1}+k_{2}+k_{3}+k_{4}
$$




$$
\begin{aligned}
\Pi_{2}= & k_{1} k_{2}+k_{1} k_{3}+k_{1} k_{4}+k_{2} k_{3}+k_{2} k_{4}+k_{3} k_{4}-\left(m_{1} n_{4}+m_{2} n_{1}+m_{3} n_{2}\right), \\
\Pi_{3}= & \left(k_{1} k_{2} k_{3}+k_{1} k_{2} k_{4}+k_{1} k_{3} k_{4}+k_{2} k_{3} k_{4}\right)+\left(m_{3} n_{2} k_{4}+m_{4} n_{3} k_{2}+m_{1} n_{4} k_{2}\right. \\
& \left.+m_{4} n_{3} k_{1}+m_{2} n_{1} k_{4}+m_{1} n_{4} k_{3}+m_{2} n_{1} k_{3}+m_{3} n_{2} k_{1}\right), \\
\Pi_{4}= & k_{1} k_{2} k_{3} k_{4}-\left(m_{4} n_{3} k_{1} k_{2}+m_{2} n_{1} k_{3} k_{4}+m_{1} n_{4} k_{2} k_{3}+m_{3} n_{2} k_{1} k_{4}\right) \\
& +m_{1} m_{3} n_{2} n_{4}+m_{2} m_{4} n_{1} n_{3}-m_{1} m_{2} m_{3} m_{4}-n_{1} n_{2} n_{3} n_{4} .
\end{aligned}
$$

Now, we will reconsider the stability of system (3.3) when $\tau=0$. According to the RouthHurwitz criterion, we have the following lemma.

Lemma 4.1 If $\tau=0$ and $\Xi_{1}>0, \Xi_{2}>0, \Xi_{3}>0, \Xi_{4}>0$, then system (3.3) is asymptotically stable.

Proof When $\tau=0$, by (4.3), we get

$$
\lambda^{4}+\Pi_{1} \lambda^{3}+\Pi_{2} \lambda^{2} \Pi_{3} \lambda+\Pi_{4}=0
$$

If the conditions of $\Xi_{1}>0, \Xi_{2}>0, \Xi_{3}>0, \Xi_{4}>0$ hold, then the roots $\lambda_{i}$ of Eq. (4.4) satisfy $\left|\arg \left(\lambda_{i}\right)\right|>\phi \pi / 2$. Thus, according to Lemma 2.1 , system (3.3) is asymptotically stable when $\tau=0$.

To throw up our main results, we further give the following assumption:

(C4) $\frac{\Upsilon_{1} \Omega_{1}+\Upsilon_{2} \Omega_{2}}{\Omega_{1}^{2}+\Omega_{2}^{2}} \neq 0$,

where

$$
\begin{aligned}
& \Upsilon_{1}=2 w_{0}\left[P_{2}^{R} \sin 2 w_{0} \tau_{0}-P_{2}^{I} \cos 2 w_{0} \tau_{0}+2\left(P_{3}^{R} \sin 4 w_{0} \tau_{0}-P_{3}^{I} \cos 4 w \tau_{0}\right)\right] \\
& \Upsilon_{2}=2 w_{0}\left[P_{2}^{R} \cos 2 w_{0} \tau_{0}+P_{2}^{I} \sin 2 w_{0} \tau_{0}+2\left(P_{3}^{R} \cos 4 \omega_{0} \tau_{0}+P_{3}^{I} \sin 4 \omega_{0} \tau_{0}\right)\right] \\
& \Omega_{1}=P_{1}^{\prime R}+\left(P_{2}^{\prime R}-2 \tau_{0} P_{2}^{R}\right) \cos 2 w_{0} \tau_{0}+\left(P_{2}^{\prime I}-2 \tau_{0} P_{2}^{I}\right) \sin 2 w_{0} \tau_{0}-4 \tau_{0} P_{3}^{R} \cos 4 \omega_{0} \tau_{0} \\
& \Omega_{2}=P_{1}^{\prime I}-\left(P_{2}^{\prime R}-2 \tau_{0} P_{2}^{R}\right) \sin 2 w_{0} \tau_{0}+\left(P_{2}^{\prime I}-2 \tau_{0} P_{2}^{I}\right) \cos 2 w_{0} \tau_{0}+4 \tau_{0} P_{3}^{R} \sin 4 w_{0} \tau_{0} .
\end{aligned}
$$

Lemma 4.2 Let $s(\tau)=v(\tau)+i w(\tau)$ be a root of Eq. (4.3) near $\tau=\tau_{j}$ satisfying $v\left(\tau_{j}\right)=0$, $w\left(\tau_{j}\right)=w_{0}$, then the following transversality condition holds:

$$
\left.\operatorname{Re}\left[\frac{d s}{d \tau}\right]\right|_{\left(w=w_{0}, \tau=\tau_{0}\right)} \neq 0
$$

Proof By using the implicit function theorem and differentiating (4.3) with respect to $\tau$, we have

$$
\begin{gathered}
P_{1}^{\prime}(s) \frac{d s}{d \tau}+P_{2}^{\prime}(s) e^{-2 s \tau} \frac{d s}{d \tau}+P_{2}(s) e^{-2 s \tau}\left(-2 \tau \frac{d s}{d \tau}-2 s\right) \\
+P_{3}^{\prime}(s) e^{-4 s \tau} \frac{d s}{d \tau}+P_{3}(s) e^{-4 s \tau}\left(-4 \tau \frac{d s}{d \tau}-4 s\right)=0
\end{gathered}
$$

where $P_{i}^{\prime}(s)$ is the derivative of $P_{i}(s)$. 
Noting that $P_{3}^{\prime}(s)=0$, therefore we have

$$
\frac{d s}{d \tau}=\frac{\Upsilon(s)}{\Omega(s)}
$$

where

$$
\begin{aligned}
& \Upsilon(s)=2 s\left[P_{2}(s) e^{-2 s \tau}+2 P_{3}(s) e^{-4 s \tau}\right] \\
& \Omega(s)=P_{1}^{\prime}(s)+\left[P_{2}^{\prime}(s)-2 \tau P_{2}(s)\right] e^{-2 s \tau}-4 \tau P_{3}(s) e^{-4 s \tau}
\end{aligned}
$$

Let $P_{i}^{\prime R}, P_{i}^{\prime I}$ be the real and imaginary parts of $P_{i}(s)(i=1,2,3)$, respectively. We further suppose that $\Upsilon_{1}, \Upsilon_{2}$ are the real and imaginary parts of $\Upsilon(s)$, respectively, and $\Omega_{1}, \Omega_{2}$ are the real and imaginary parts of $\Omega(s)$, respectively, then

$$
\left.\operatorname{Re}\left[\frac{d s}{d \tau}\right]\right|_{\left(\tau=\tau_{0}^{*}, w=w_{0}^{*}\right)}=\frac{\Upsilon_{1} \Omega_{1}+\Upsilon_{2} \Omega_{2}}{\Omega_{1}^{2}+\Omega_{2}^{2}}
$$

And from (C3) we conclude that the transversality condition is satisfied. This completes the proof of Lemma 4.2.

From the above analysis, we can obtain the following results.

Theorem 4.1 If system (3.3) satisfies:

(1) Under assumptions (C1)-(C4), then the zero equilibrium point is globally asymptotically stable for $\tau \in[0,+\infty)$.

(2) Under assumptions (C1), (C3), and (C4), then

(i) the zero equilibrium point is locally asymptotically stable for $\tau \in\left[0, \tau_{0}\right)$;

(ii) system (3.3) undergoes a Hopf bifurcation at the origin when $\tau=\tau_{0}$. That is, $a$ family of periodic solutions can bifurcate from the zero equilibrium point at $\tau=\tau_{0}$.

Theorem 4.1 shows that there is an explicit communication time delay value $\tau=\tau_{0}$, which can determine the stability of system (3.3) and can induce oscillatory dynamics even when the deterministic counterpart of system (3.3) exhibits no oscillations.

\subsection{Bifurcation analysis involving leakage delays}

In this subsection, we first study the stability of system (3.2) by taking the leakage time delay as the bifurcation parameter. Then we further look for the sufficient conditions of Hopf bifurcation for the proposed system.

It is obvious that the origin is an equilibrium point of system (3.2) under assumption (C1). The linear equation of system (3.2) at the origin is

$$
\left\{\begin{array}{l}
D^{\phi} x_{1}(t)=-r_{1} x_{1}(t-\tau)+\mu_{1} x_{1}(t)+m_{1} x_{4}(t-\tau)+n_{1} x_{2}(t-\tau), \\
D^{\phi} x_{2}(t)=-r_{2} x_{2}(t-\tau)+\mu_{2} x_{2}(t)+m_{2} x_{1}(t-\tau)+n_{2} x_{3}(t-\tau), \\
D^{\phi} x_{3}(t)=-r_{3} x_{3}(t-\tau)+\mu_{3} x_{3}(t)+m_{3} x_{2}(t-\tau)+n_{3} x_{4}(t-\tau), \\
D^{\phi} x_{4}(t)=-r_{4} x_{4}(t-\tau)+\mu_{4} x_{4}(t)+m_{4} x_{3}(t-\tau)+n_{4} x_{1}(t-\tau),
\end{array}\right.
$$


and the associated characteristic equation of system (4.13) is

$$
\operatorname{det}\left(\begin{array}{cccc}
s^{\phi}-\mu+r_{1} e^{-s \tau} & -n_{1} e^{-s \tau} & 0 & -m_{1} e^{-s \tau} \\
-m_{2} e^{-s \tau} & s^{\phi}-\mu+r_{2} e^{-s \tau} & -n_{2} e^{-s \tau} & 0 \\
0 & -m_{3} e^{-s \tau} & s^{\phi}-\mu+r_{3} e^{-s \tau} & -n_{3} e^{-s \tau} \\
-n_{4} e^{-s \tau} & 0 & -m_{4} e^{-s \tau} & s^{\phi}-\mu+r_{4} e^{-s \tau}
\end{array}\right)=0
$$

which equals to the following equation:

$$
\begin{gathered}
\left(s^{\phi}-\mu\right)^{4}+Q_{1}\left(s^{\phi}-\mu\right)^{3} e^{-s \tau}+Q_{2}\left(s^{\phi}-\mu\right)^{2} e^{-2 s \tau} \\
+Q_{3}\left(s^{\phi}-\mu\right) e^{-3 s \tau}+Q_{4} e^{-4 s \tau}=0,
\end{gathered}
$$

in which

$$
\begin{aligned}
Q_{1}= & r_{1}+r_{2}+r_{3}+r_{4}, \\
Q_{2}= & -n_{3} m_{4}+r_{2} r_{4}-m_{3} n_{2}+r_{1} r_{4}+r_{1} r_{3}+r_{2} r_{3}-m_{2} n_{1}+r_{3} r_{4}-m_{1} n_{4}+r_{1} r_{2}, \\
Q_{3}= & -m_{3} n_{2} r_{4}+r_{1} r_{3} r_{4}+r_{2} r_{3} r_{4}-r_{2} n_{3} m_{4}+r_{1} r_{2} r_{3}-m_{2} n_{1} r_{4}-r_{1} n_{3} m_{4}+r_{1} r_{2} r_{4} \\
& -n_{4} m_{1} r_{3}-m_{3} n_{2} r_{1}, \\
Q_{4}= & r_{1} r_{2} r_{3} r_{4}-m_{1} m_{2} m_{3} m_{4}-n_{1} n_{2} n_{3} n_{4}-m_{2} n_{1} r_{3} r_{4}-m_{3} n_{2} r_{1} r_{4}+r_{1} r_{2} r_{4}-n_{4} m_{1} r_{3} \\
& -m_{3} n_{2} r_{1}-m_{1} n_{4} r_{2} r_{3}+m_{1} m_{3} n_{2} n_{4}-m_{3} m_{4} r_{1} r_{2}+m_{2} m_{4} n_{1} n_{3} .
\end{aligned}
$$

Multiplying $e^{4 s \tau}$ on both sides of Eq. (4.15), we get

$$
\left[\left(s^{\phi}-u\right) e^{s \tau}\right]^{4}+Q_{1}\left[\left(s^{\phi}-u\right) e^{s \tau}\right]^{3}+Q_{2}\left[\left(s^{\phi}-u\right) e^{s \tau}\right]^{2}+Q_{3}\left(s^{\phi}-u\right) e^{s \tau}+Q_{4}=0 .
$$

Suppose that $h+i k=\left(s^{\phi}-u\right) e^{s \tau}$ in Eq. (4.16), it follows that

$$
(h+i k)^{4}+Q_{1}(h+i k)^{3}+Q_{2}(h+i k)^{2}+Q_{3}(h+i k)+Q_{4}=0 .
$$

Since $Q_{i}$ are constants, for all the roots $(h+i k)$ of Eq. (4.17), the details can be seen in [38]. $s=i \omega=\omega\left(\cos \frac{\pi}{2}+i \sin \frac{\pi}{2}\right)(\omega>0)$ is a purely imaginary root of Eq. (4.17) if and only if

$$
\left\{\begin{array}{l}
\left(\omega^{\phi} \cos \frac{\phi \pi}{2}-u\right) \cos \omega \tau-\omega^{\phi} \sin \frac{\phi \pi}{2} \sin \omega \tau=h, \\
\left(\omega^{\phi} \cos \frac{\phi \pi}{2}-u\right) \sin \omega \tau-\omega^{\phi} \sin \frac{\phi \pi}{2} \cos \omega \tau=k .
\end{array}\right.
$$

If $\omega^{2 \phi}-2 u \omega^{\phi} \cos \frac{\phi \pi}{2}+u^{2} \neq 0$, then by Eq. (4.18) we have that

$$
\left\{\begin{array}{l}
\cos \omega \tau=\frac{\omega^{\phi}\left(h \cos \frac{\phi \pi}{2}+k \sin \frac{\phi \pi}{2}\right)-h u}{\omega^{2 \phi}-2 u \omega^{\phi} \cos \frac{\phi \pi}{2}+u^{2}} \\
\sin \omega \tau=\frac{\omega^{\phi}\left(k \cos \frac{\phi \pi}{2}-h \sin \frac{\phi \pi}{2}\right)-k u}{\omega^{2 \phi}-2 u \omega^{\phi} \cos \frac{\phi \pi}{2}+u^{2}}
\end{array}\right.
$$

Because $\sin ^{2} \omega \tau+\cos ^{2} \omega \tau=1$, Eq. (4.19) implies that

$$
\omega^{2 \phi}=h^{2}+k^{2}
$$


By a direct computation, one can have

$$
\omega=\sqrt[2 \phi]{h^{2}+k^{2}}
$$

According to $\cos \omega \tau=\frac{\omega^{\phi}\left(h \cos \frac{\phi \pi}{2}+k \sin \frac{\phi \pi}{2}\right)-h u}{\omega^{2 \phi}-2 u \omega^{\phi} \cos \frac{\phi \pi}{2}+u^{2}}$, we obtain that

$$
\tau^{(l)}=\frac{1}{\omega}\left[\arccos \left(\frac{\omega^{\phi}\left(h \cos \frac{\phi \pi}{2}+k \sin \frac{\phi \pi}{2}\right)-h u}{\omega^{2 \phi}-2 u \omega^{\phi} \cos \frac{\phi \pi}{2}+u^{2}}\right)+2 l \pi\right], \quad l=0,1,2, \ldots
$$

To establish the main results for system (3.2), it is necessary to make the following assumptions.

(C5) Eq. (4.21) has at least one positive real root.

For system (3.2), we define the bifurcation point as follows:

$$
\tau_{0}=\min \left\{\tau^{(l)}\right\}, \quad l=0,1,2, \ldots
$$

where $\tau^{(l)}$ is defined by (4.22).

To produce our main results, furthermore, we assume that the following condition holds:

(C6) $\frac{\Phi_{1} \Psi_{1}+\Phi_{2} \Psi_{2}}{\Psi_{1}^{2}+\Psi_{2}^{2}} \neq 0$,

where

$$
\begin{aligned}
\Phi_{1}= & \omega_{0}\left[m_{2}^{R} \sin \omega_{0} \tau_{0}^{*}-m_{2}^{I} \cos \omega_{0} \tau_{0}^{*}+2\left(m_{3}^{R} \sin 2 \omega_{0} \tau_{0}^{*}-m_{3}^{I} \cos 2 \omega_{0} \tau_{0}^{*}\right)\right] \\
& \left.\left.+3\left(m_{4}^{R} \sin 3 \omega_{0} \tau_{0}^{*}-m_{4}^{I} \cos 3 \omega_{0} \tau_{0}^{*}\right)\right]+4 m_{5}^{R} \sin 4 \omega_{0} \tau_{0}^{*}\right], \\
\Phi_{2}= & \omega_{0}\left[m_{2}^{R} \cos \omega_{0} \tau_{0}^{*}+m_{2}^{I} \sin \omega_{0} \tau_{0}^{*}+2\left(m_{3}^{R} \cos 2 \omega_{0} \tau_{0}^{*}+m_{3}^{I} \sin 2 \omega_{0} \tau_{0}^{*}\right)\right] \\
& \left.\left.+3\left(m_{4}^{R} \cos 3 \omega_{0} \tau_{0}^{*}+m_{4}^{I} \sin 3 \omega_{0} \tau_{0}^{*}\right)\right]+4 m_{5}^{R} \cos 4 \omega_{0} \tau_{0}^{*}\right], \\
\Psi_{1}= & m_{1}^{\prime R}+\left(m_{2}^{\prime R}-\tau_{0}^{*} m_{2}^{R}\right) \cos \omega_{0} \tau_{0}^{*}+\left(m_{2}^{\prime I}-\tau_{0}^{*} m_{2}^{I}\right) \sin \omega_{0} \tau_{0}^{*} \\
& +\left(m_{3}^{\prime R}-2 \tau_{0}^{*} m_{3}^{R}\right) \cos 2 \omega_{0} \tau_{0}^{*}+\left(m_{3}^{\prime I}-2 \tau_{0}^{*} m_{3}^{I}\right) \sin 2 \omega_{0} \tau_{0}^{*} \\
& +\left(m_{4}^{\prime R}-3 \tau_{0}^{*} m_{4}^{R}\right) \cos 3 \omega_{0} \tau_{0}^{*}+\left(m_{4}^{\prime I}-3 \tau_{0}^{*} m_{4}^{I}\right) \sin 3 \omega_{0} \tau_{0}^{*} \\
& -4 \tau_{0}^{*} m_{5}^{R} \cos 4 \omega_{0} \tau_{0}^{*}, \\
\Psi_{2}= & m_{1}^{\prime I}-\left(m_{2}^{\prime R}-\tau_{0}^{*} m_{2}^{R}\right) \sin \omega_{0} \tau_{0}^{*}+\left(m_{2}^{\prime I}-\tau_{0}^{*} m_{2}^{I}\right) \cos \omega_{0} \tau_{0}^{*} \\
& -\left(m_{3}^{\prime R}-2 \tau_{0}^{*} m_{3}^{R}\right) \sin 2 \omega_{0} \tau_{0}+\left(m_{3}^{\prime I}-2 \tau_{0}^{*} m_{3}^{I}\right) \cos 2 \omega_{0} \tau_{0}^{*} \\
& -\left(m_{4}^{\prime R}-3 \tau_{0}^{*} m_{4}^{R}\right) \sin 3 \omega_{0} \tau_{0}^{*}+\left(m_{4}^{\prime I}-3 \tau_{0}^{*} m_{4}^{I}\right) \cos 3 \omega_{0} \tau_{0}^{*} \\
& +4 \tau_{0}^{*} m_{5}^{R} \sin 4 \omega_{0} \tau_{0}^{*} .
\end{aligned}
$$

Lemma 4.3 Let $s(\tau)=\mu(\tau)+i \omega(\tau)$ be a root of system (3.2) near $\tau=\tau_{j}$ satisfying $\mu\left(\tau_{j}\right)=0$, $\omega\left(\tau_{j}\right)=\omega_{0}$, then the following transversality condition holds:

$$
\left.\operatorname{Re}\left[\frac{d s}{d \tau}\right]\right|_{\left(\omega=\omega_{0}, \tau=\tau_{0}^{*}\right)} \neq 0
$$


Proof Equation (4.15) can be transformed into

$$
m_{1}(s)+m_{2}(s) e^{-s \tau}+m_{3}(s) e^{-2 s \tau}+m_{4}(s) e^{-3 s \tau}+m_{5}(s) e^{-4 s \tau}=0,
$$

where $m_{1}(s)=\left(s^{\alpha}-\mu\right)^{4}, m_{2}(s)=Q_{1}\left(s^{\alpha}-\mu\right)^{3}, m_{3}(s)=Q_{2}\left(s^{\alpha}-\mu\right)^{2}, m_{4}(s)=Q_{3}\left(s^{\alpha}-\mu\right), m_{5}(s)=$ $Q_{4}$.

Based on the implicit function theorem and differentiating (4.23) with respect to $\tau$, it reads

$$
\begin{gathered}
m_{1}^{\prime}(s) \frac{d s}{d \tau}+m_{2}^{\prime}(s) e^{-s \tau} \frac{d s}{d \tau}+m_{2}(s) e^{-s \tau}\left(-\tau \frac{d s}{d \tau}-s\right) \\
+m_{3}^{\prime}(s) e^{-2 s \tau} \frac{d s}{d \tau}+m_{3}(s) e^{-2 s \tau}\left(-2 \tau \frac{d s}{d \tau}-2 s\right) \\
+m_{4}^{\prime}(s) e^{-3 s \tau} \frac{d s}{d \tau}+m_{4}(s) e^{-3 s \tau}\left(-3 \tau \frac{d s}{d \tau}-3 s\right) \\
+m_{5}^{\prime}(s) e^{-4 s \tau} \frac{d s}{d \tau}+m_{5}(s) e^{-4 s \tau}\left(-4 \tau \frac{d s}{d \tau}-4 s\right)=0
\end{gathered}
$$

where $m_{i}^{\prime}(s)$ are the derivatives of $m_{i}(s)$.

Based on Eq. (4.23) and $m_{5}^{\prime}(s)=0$, one can have

$$
\frac{d s}{d \tau}=\frac{\Phi(s)}{\Psi(s)}
$$

where

$$
\begin{aligned}
\Phi(s)= & \left.s\left[m_{2}(s) e^{-s \tau}+2 m_{3}(s) e^{-2 s \tau}+3 m_{4}(s) e^{-3 s \tau}+4 m_{5}(s)\right) e^{-4 s \tau}\right], \\
\Psi(s)= & m_{1}^{\prime}(s)+\left[m_{2}^{\prime}(s)-\tau m_{2}(s)\right] e^{-s \tau}+\left[m_{3}^{\prime}(s)-2 \tau m_{3}(s)\right] e^{-2 s \tau} \\
& +\left[m_{4}^{\prime}(s)-3 \tau m_{4}(s)\right] e^{-3 s \tau}-4 \tau m_{5}(s) e^{-4 s \tau} .
\end{aligned}
$$

Let $m_{i}^{\prime R}, m^{\prime I}$ be the real and imaginary parts of $m_{i}(s)(i=1,2,3)$, respectively; $\Phi_{1}, \Phi_{2}$ be the real and imaginary parts of $\Phi(s)$, respectively; and $\Psi_{1}, \Psi_{2}$ be the real and imaginary parts of $\Psi(s)$, respectively, then it can be derived from (4.25) that

$$
\left.\operatorname{Re}\left[\frac{d s}{d \tau}\right]\right|_{\left(\tau=\omega=\omega_{0}, \tau_{0}^{*}\right)}=\frac{\Phi_{1} \Psi_{1}+\Phi_{2} \Psi_{2}}{\Psi_{1}^{2}+\Psi_{2}^{2}}
$$

From (C6), we can conclude that the transversality condition is met.

Assume that (C1), (C5)-(C6), Lemma 2.1, and Lemma 4.3 hold, we can obtain the following theorem.

Theorem 4.2 For system (3.2), the following results hold:

(1) If (C1) and (C5) are satisfied, then the zero equilibrium point is globally asymptotically stable for $\tau \in[0,+\infty)$.

(2) If (C1), (C5)-(C6) hold, then

(i) the zero equilibrium point is locally asymptotically stable for $\tau \in\left[0, \tau_{0}^{*}\right)$; 

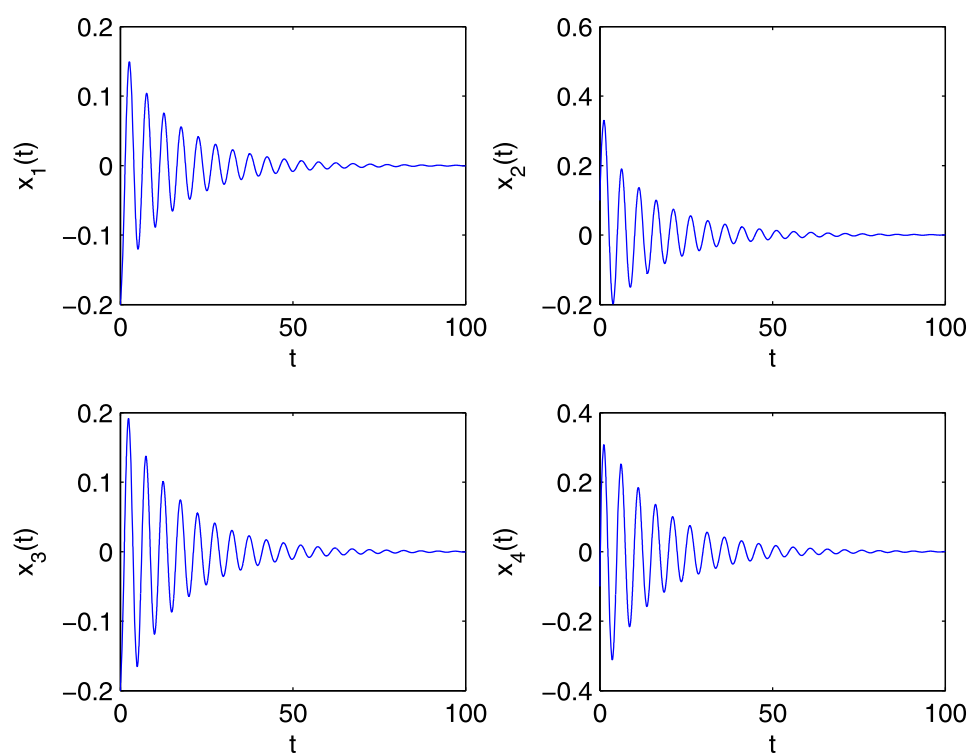

Figure 1 Time responses of system (5.1) with $\phi=0.92, \tau=0.47<\tau_{0}=0.5421$
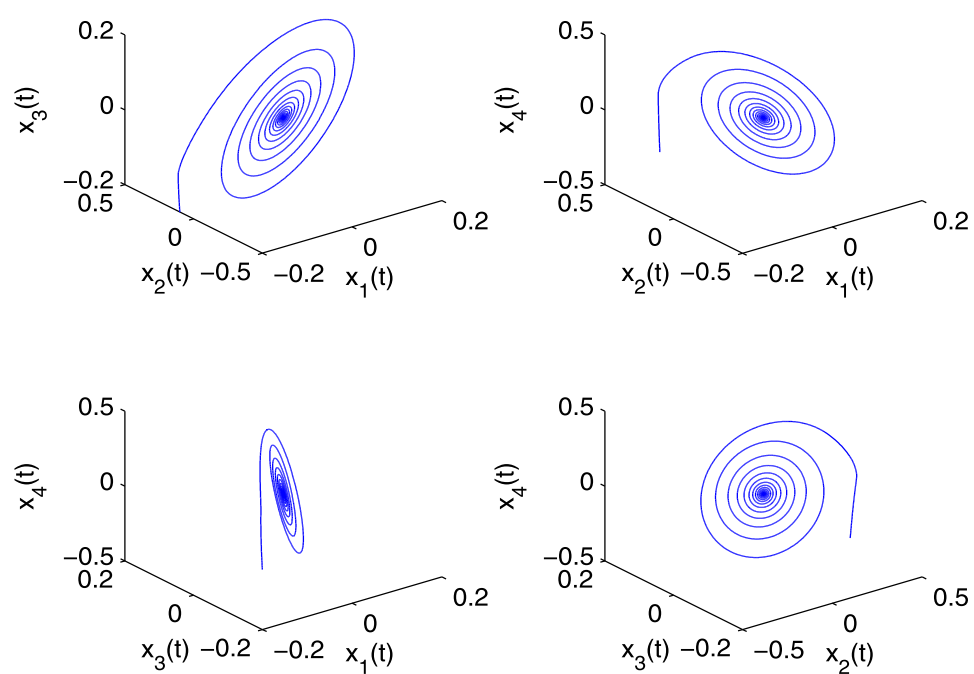

Figure 2 Phase diagrams of system (5.1) with $\phi=0.92, \tau=0.47<\tau_{0}=0.5421$

(ii) system (3.2) undergoes a Hopf bifurcation at the origin when $\tau=\tau_{0}^{*}$, i.e., it has one branch of periodic solutions bifurcating from the zero equilibrium point near $\tau=\tau_{0}^{*}$.

This theorem demonstrates that the stability and the Hopf bifurcation of the neural network are not only crucially dependent on the communication delays, but also heavily influenced by the leakage delay. It is therefore essential for considering the effects of communication and leakage delays in designing or controlling neural networks. 

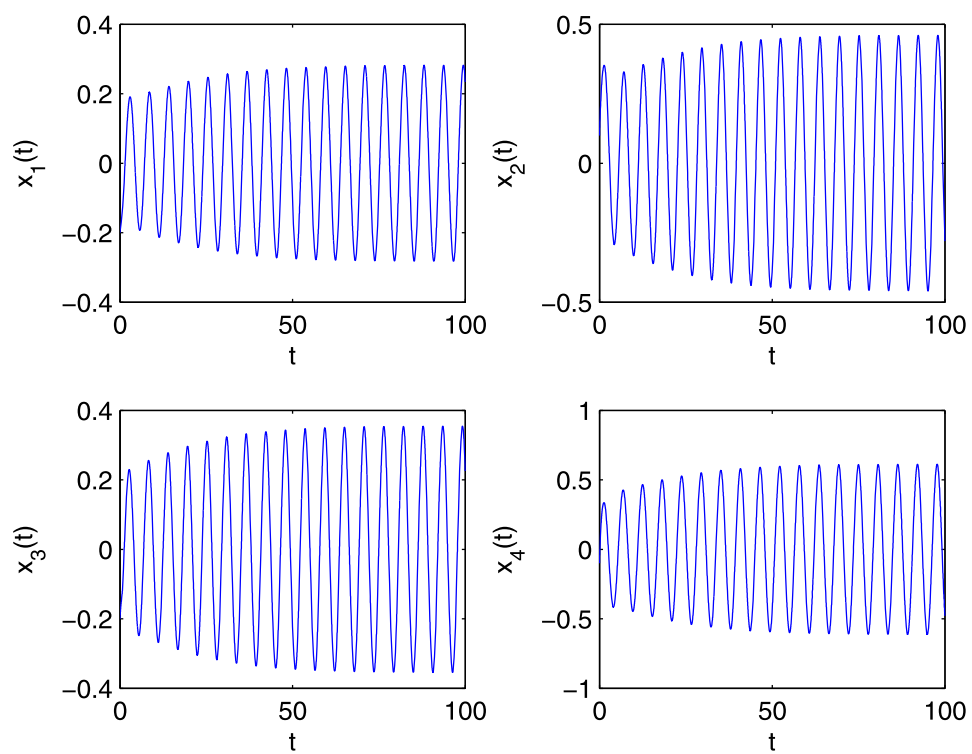

Figure 3 Time responses of system (5.1) with $\phi=0.92, \tau=0.6>\tau_{0}=0.5421$
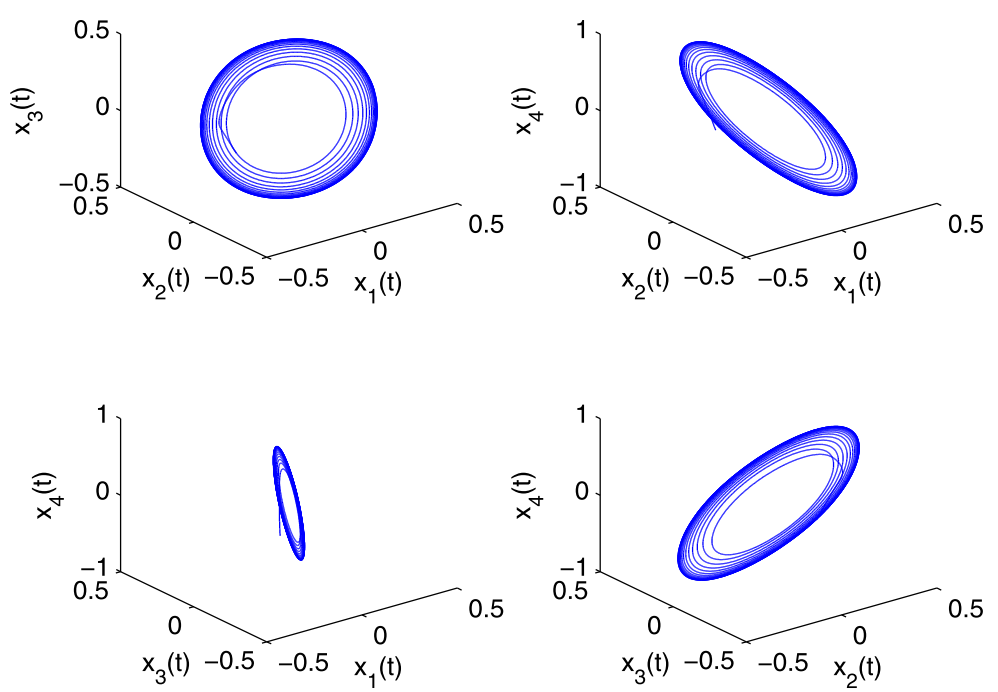

Figure 4 Phase diagrams of system (5.1) with $\phi=0.92, \tau=0.6>\tau_{0}=0.5421$

\section{Illustrative examples}

In this section, we give several examples to show the feasibility and effectiveness of the results obtained in this paper. All of the simulation results are based on Adama-BashforthMoulton predictor-corrector scheme [40] with step-length $h=0.01$. 

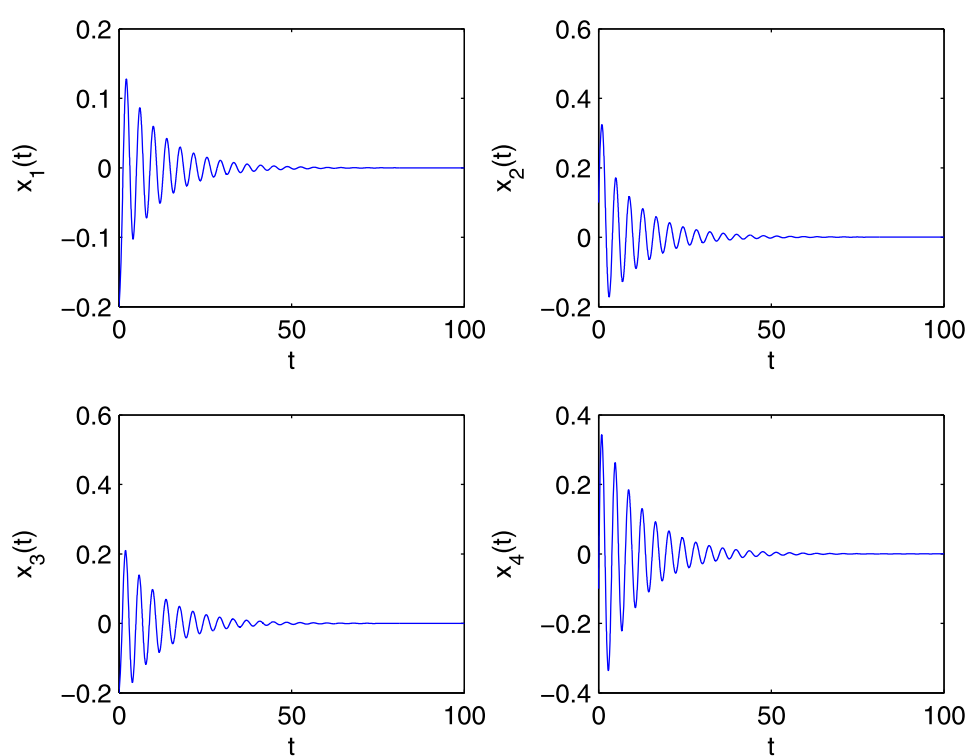

Figure 5 Time responses of system (5.2) with $\phi=0.92, \tau=0.36<\tau_{0}^{*}=0.3976$
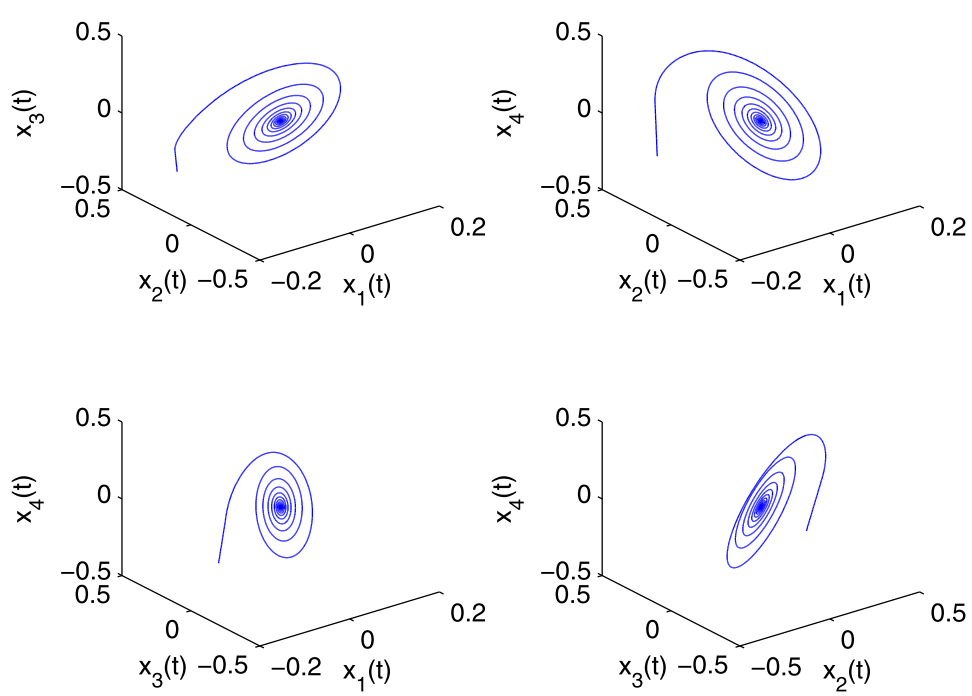

Figure 6 Phase diagrams of system (5.2) with $\phi=0.92, \tau=0.36<\tau_{0}^{*}=0.3976$

\subsection{Example 1}

Consider the following system without leakage delays:

$$
\left\{\begin{array}{l}
D^{\phi} x_{1}(t)=-0.2 x_{1}(t)-0.2 \tanh \left(x_{1}(t)\right)+0.3 \tanh \left(x_{4}(t-\tau)\right)+0.4 \tanh \left(x_{2}(t-\tau)\right), \\
D^{\phi} x_{2}(t)=0.4 x_{2}(t)-0.2 \tanh \left(x_{2}(t)\right)+1.2 \tanh \left(x_{1}(t-\tau)\right)-0.8 \tanh \left(x_{3}(t-\tau)\right), \\
D^{\phi} x_{3}(t)=0.6 x_{3}(t)-0.2 \tanh \left(x_{3}(t)\right)+0.4 \tanh \left(x_{2}(t-\tau)\right)+0.6 \tanh \left(x_{4}(t-\tau)\right), \\
D^{\phi} x_{4}(t)=0.8 x_{4}(t)-0.2 \tanh \left(x_{4}(t)\right)-1.6 \tanh \left(x_{3}(t-\tau)\right)-1.5 \tanh \left(x_{1}(t-\tau)\right) .
\end{array}\right.
$$



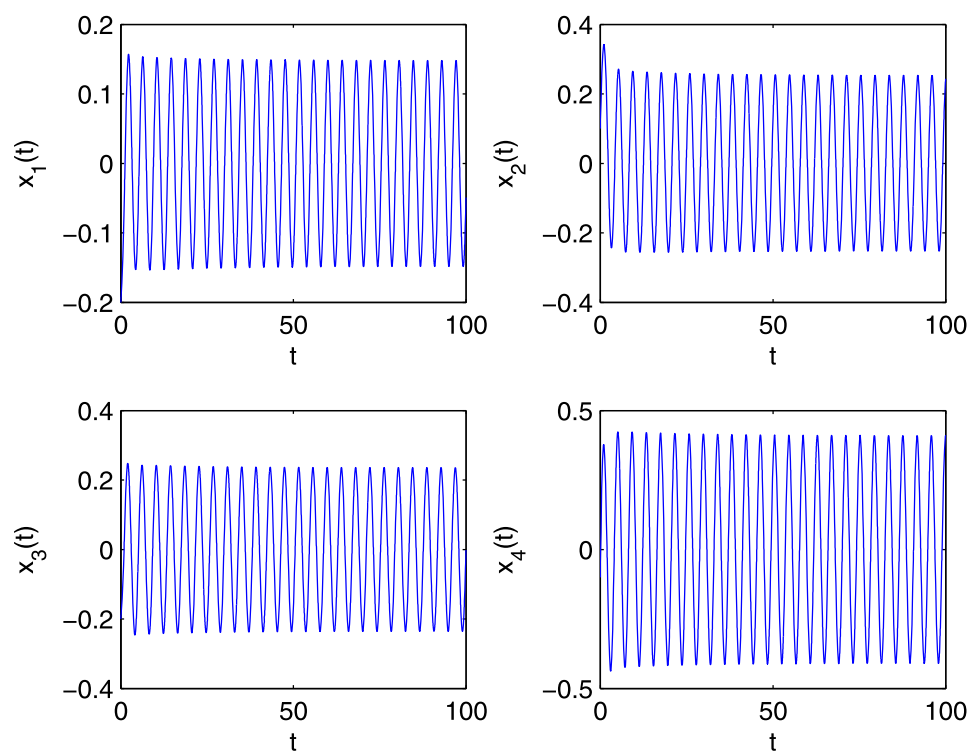

Figure 7 Time responses of system (5.2) with $\phi=0.92, \tau=0.42>\tau_{0}^{*}=0.3976$
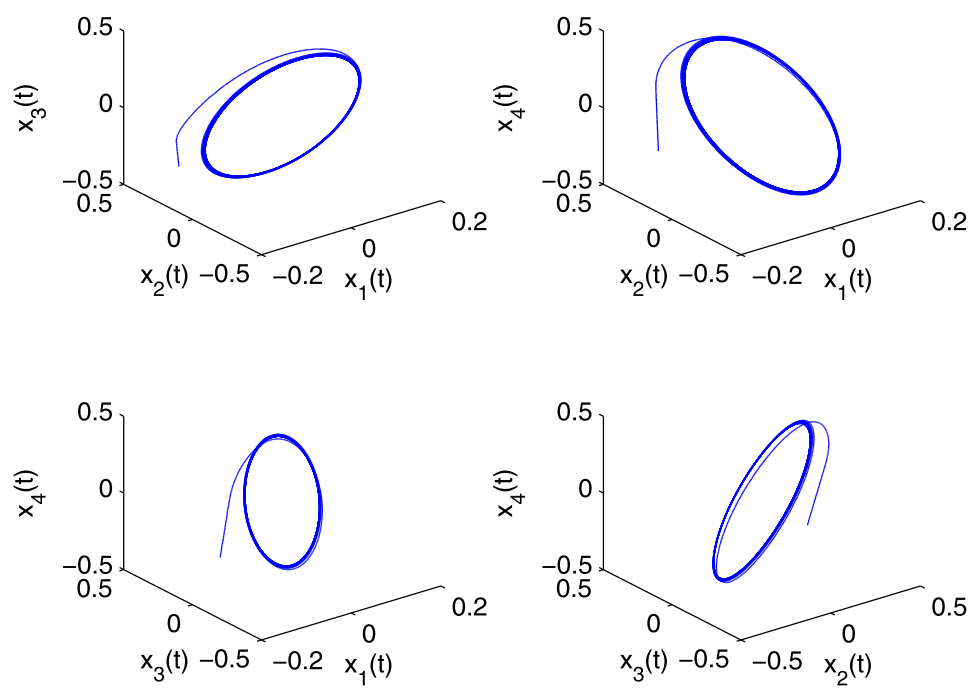

Figure 8 Phase diagrams of system (5.2) with $\phi=0.92, \tau=0.42>\tau_{0}^{*}=0.3976$

In this case, let $\phi=0.92$, and the initial values are selected as $\left(x_{1}(0), x_{2}(0), y_{1}(0), y_{2}(0)\right)=$ $(-0.2,0.1,-0.2,-0.1)$. By computing, we get $\omega_{0}=1.1675$, and then $\tau_{0}=0.5421$. Obviously, system (5.1) at the zero equilibrium point is locally asymptotically stable when $\tau=0.47<$ $\tau_{0}$, as shown in Figs. 1-2. Furthermore, Figs. $3-4$ display that the zero equilibrium point of system (5.1) is unstable, and Hopf bifurcation occurs when $\tau=0.6>\tau_{0}$. 


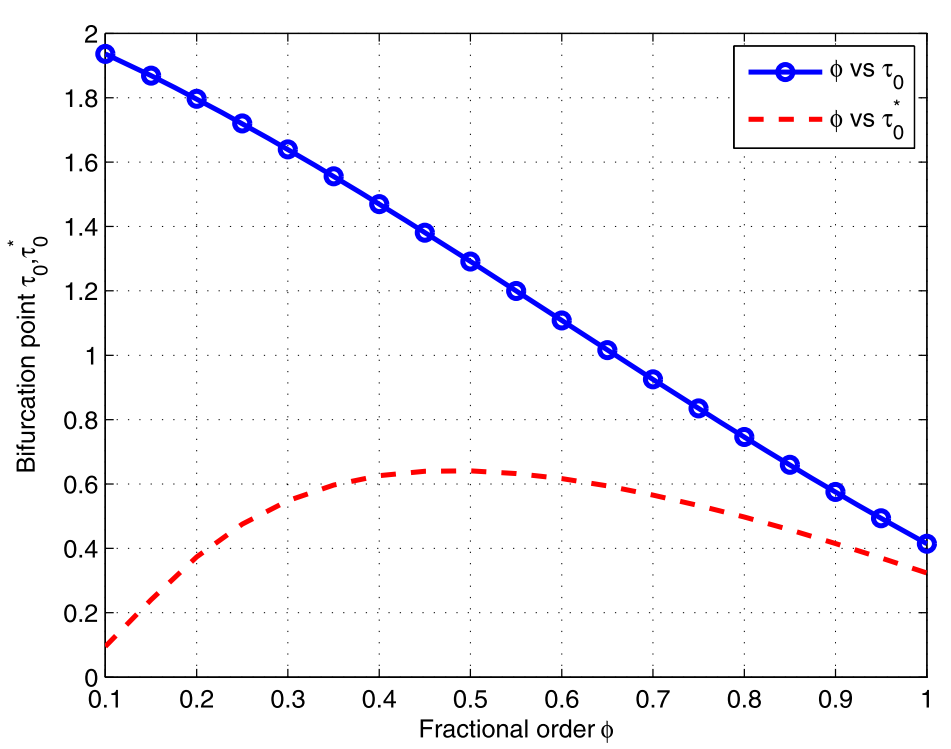

Figure 9 Effects of $\phi$ on $\tau_{0}$ in system (5.1) and $\tau_{0}^{*}$ in system (5.2)
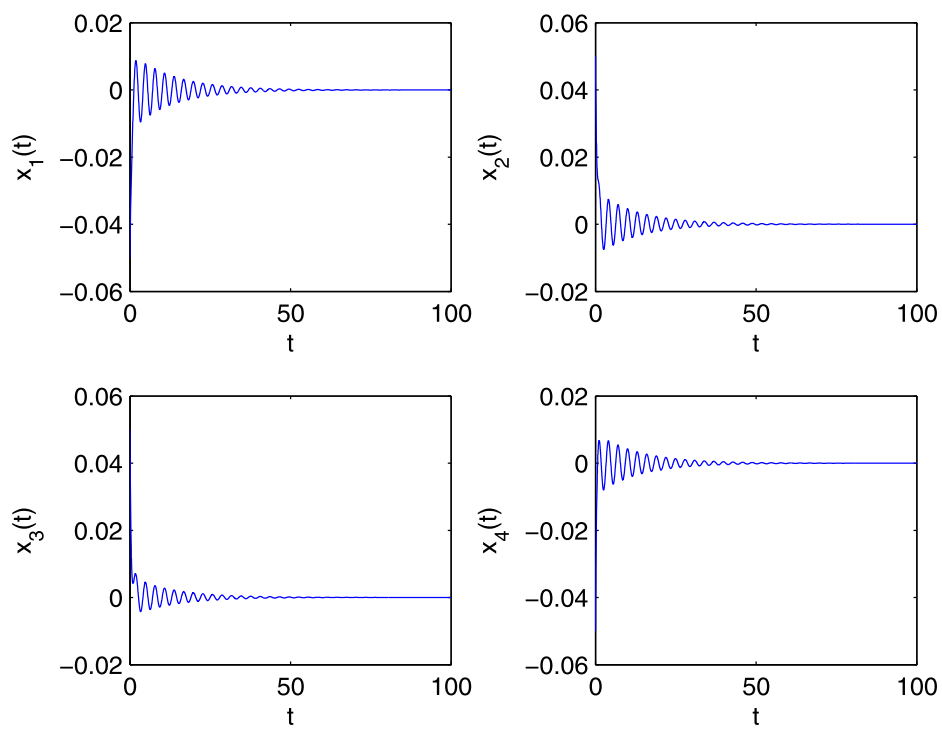

Figure 10 State response of system (5.3) with $\phi=0.96, \tau=0.38<\tau_{0}=0.4486$

\subsection{Example 2}

For making a comparison with Example 1, let $\phi=0.92$, and now we consider the following system with leakage delays:

$$
\left\{\begin{array}{l}
D^{\phi} x_{1}(t)=-0.2 x_{1}(t-\tau)-0.2 \tanh \left(x_{1}(t)\right)+0.3 \tanh \left(x_{4}(t-\tau)\right)+0.4 \tanh \left(x_{2}(t-\tau)\right), \\
D^{\phi} x_{2}(t)=0.4 x_{2}(t-\tau)-0.2 \tanh \left(x_{2}(t)\right)+1.2 \tanh \left(x_{1}(t-\tau)\right)-0.8 \tanh \left(x_{3}(t-\tau)\right), \\
D^{\phi} x_{3}(t)=0.6 x_{3}(t-\tau)-0.2 \tanh \left(x_{3}(t)\right)+0.4 \tanh \left(x_{2}(t-\tau)\right)+0.6 \tanh \left(x_{4}(t-\tau)\right), \\
D^{\phi} x_{4}(t)=0.8 x_{4}(t-\tau)-0.2 \tanh \left(x_{4}(t)\right)-1.6 \tanh \left(x_{3}(t-\tau)\right)-1.5 \tanh \left(x_{1}(t-\tau)\right) .
\end{array}\right.
$$



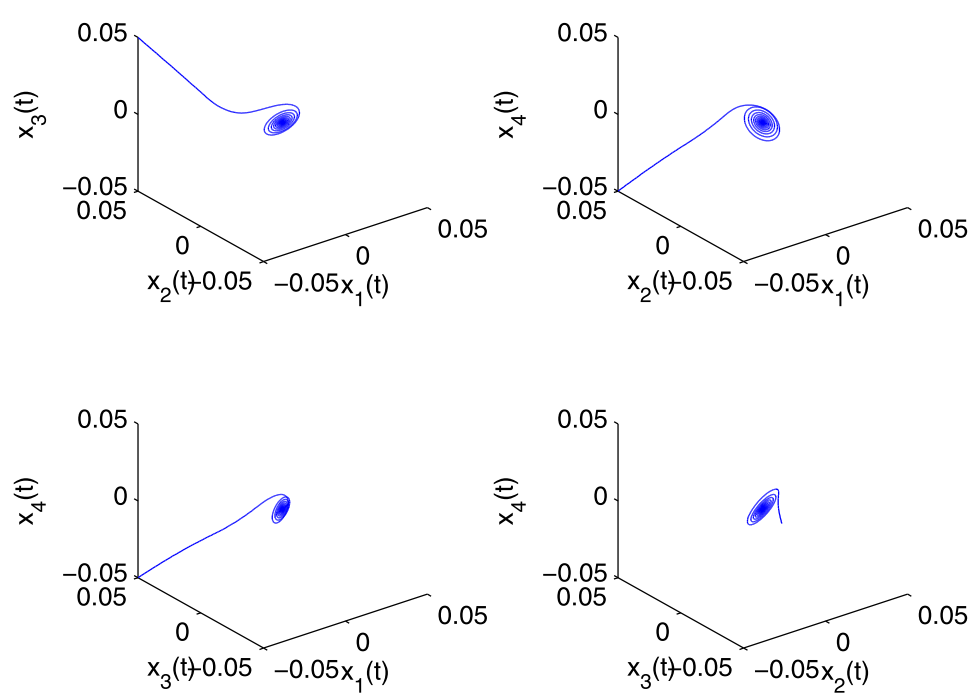

Figure 11 Phase portrait of system (5.3) with $\phi=0.96, \tau=0.38<\tau_{0}=0.4486$
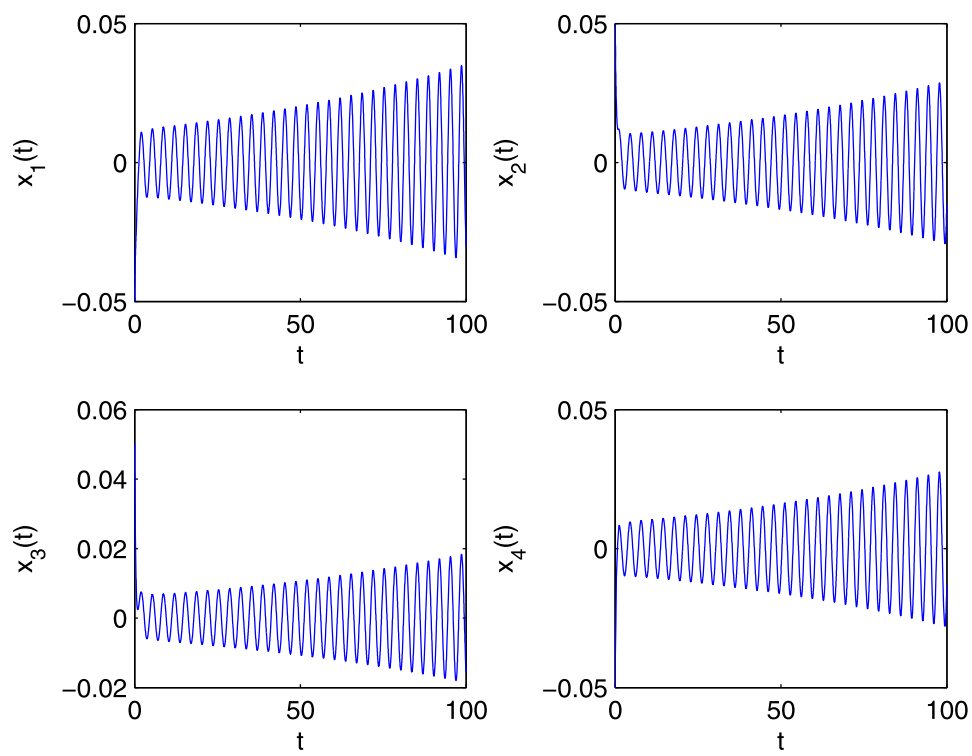

Figure 12 State response of system (5.3) with $\phi=0.96, \tau=0.46>\tau_{0}=0.4486$

By a simple calculation, we have $\omega_{0}=1.5554, \tau_{0}^{*}=0.3976$. Therefore, the zero equilibrium point of system (5.2) is locally asymptotically stable when $\tau=0.36<\tau_{0}$, as described in Figs. 5-6. Furthermore, the zero equilibrium point of system (5.2) is unstable, and Hopf bifurcation occurs when $\tau=0.42>\tau_{0}^{*}$, as depicted in Figs. 7-8.

To better reflect the impact of leakage delay on the bifurcation point for system (5.2), the corresponding bifurcation point $\tau_{0}, \tau_{0}^{*}$ can be determined as the order $\phi$ varies. It can be seen from Fig. 9 that the values of $\tau_{0}$ are larger than the case of $\tau_{0}^{*}$ for the same order $\phi$. This implies that Hopf bifurcation easily occurs in advance for system (5.2) involving leakage delay compared with system (5.1) for some fixed order $\phi$. 

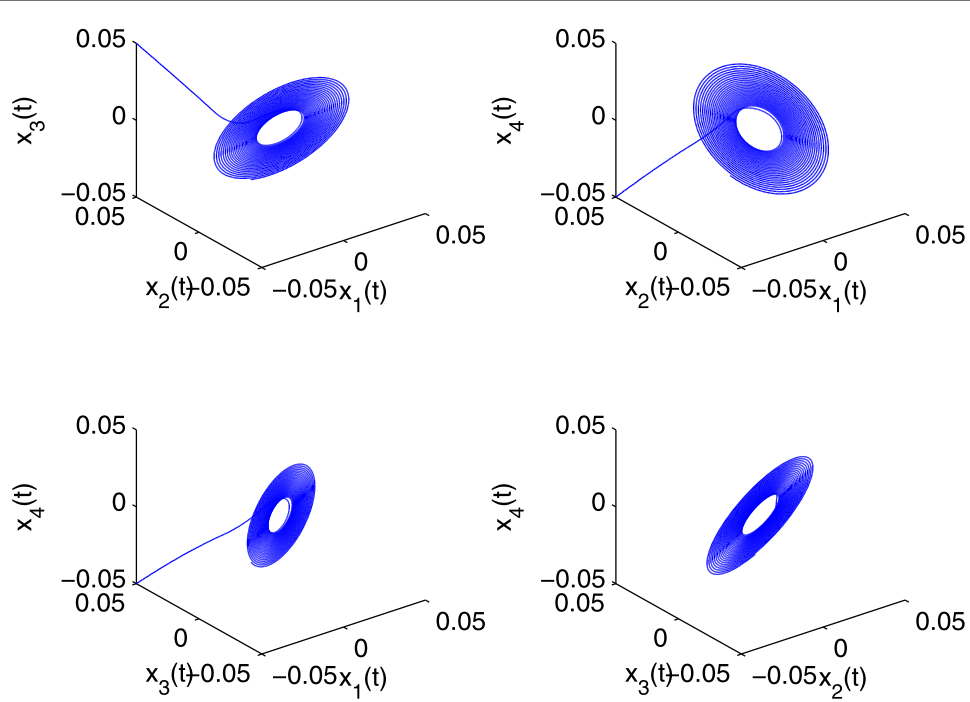

Figure 13 Phase portrait of system (5.3) with $\phi=0.96, \tau=0.46>\tau_{0}=0.4486$
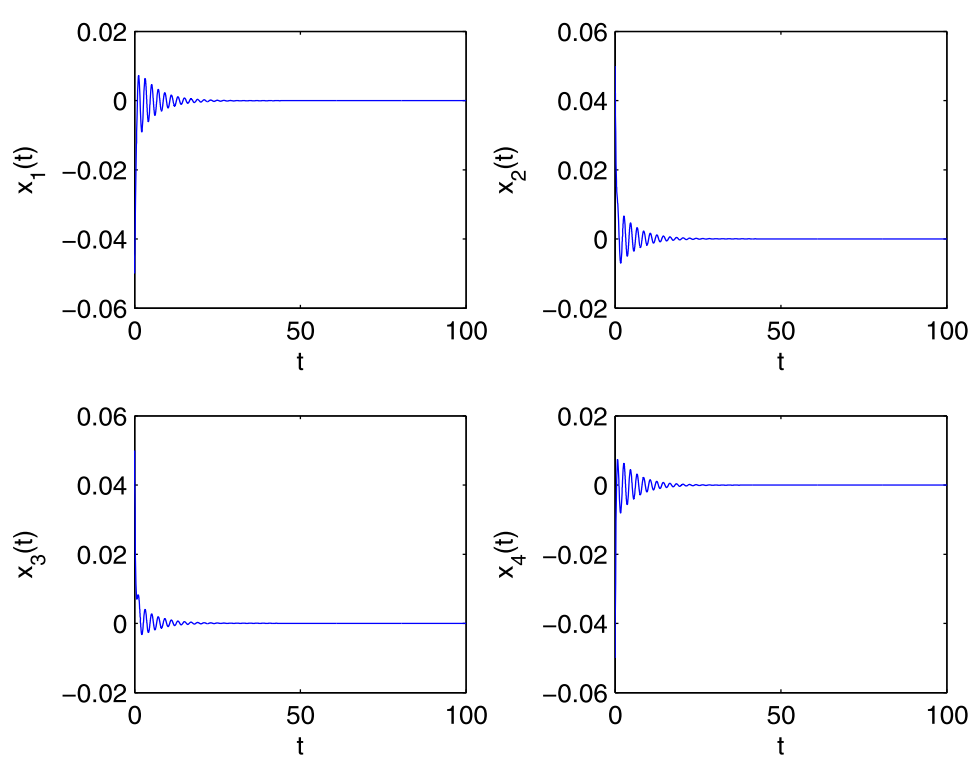

Figure 14 State response of system (5.4) with $\phi=0.96, \tau=0.22<\tau_{0}=0.2427$

\subsection{Example 3}

Consider the following system without leakage delays:

$$
\left\{\begin{array}{l}
D^{\phi} x_{1}(t)=x_{1}(t)-0.8 \tanh \left(x_{1}(t)\right)+1.8 \tanh \left(x_{4}(t-\tau)\right)+1.5 \tanh \left(x_{2}(t-\tau)\right) \\
D^{\phi} x_{2}(t)=1.2 x_{2}(t)-0.8 \tanh \left(x_{2}(t)\right)-1.5 \tanh \left(x_{1}(t-\tau)\right)-1.6 \tanh \left(x_{3}(t-\tau)\right) \\
D^{\phi} x_{3}(t)=0.9 x_{3}(t)-0.8 \tanh \left(x_{3}(t)\right)+0.5 \tanh \left(x_{2}(t-\tau)\right)+1.2 \tanh \left(x_{4}(t-\tau)\right) \\
D^{\phi} x_{4}(t)=1.5 x_{4}(t)-0.8 \tanh \left(x_{4}(t)\right)-1.2 \tanh \left(x_{3}(t-\tau)\right)-1.8 \tanh \left(x_{1}(t-\tau)\right)
\end{array}\right.
$$



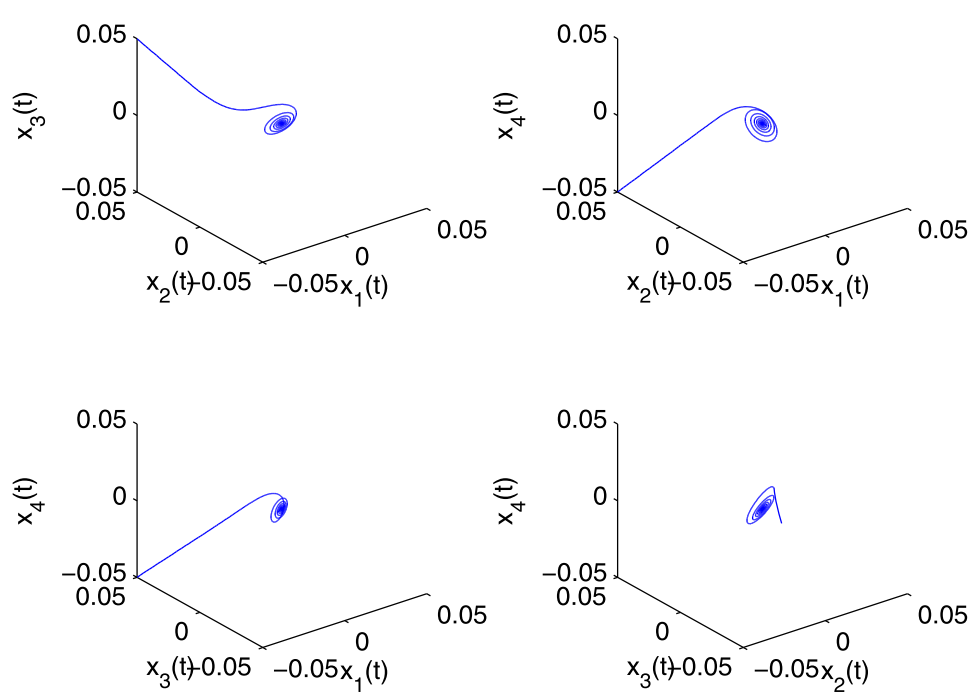

Figure 15 Phase portrait of system (5.4) with $\phi=0.96, \tau=0.22<\tau_{0}=0.2427$
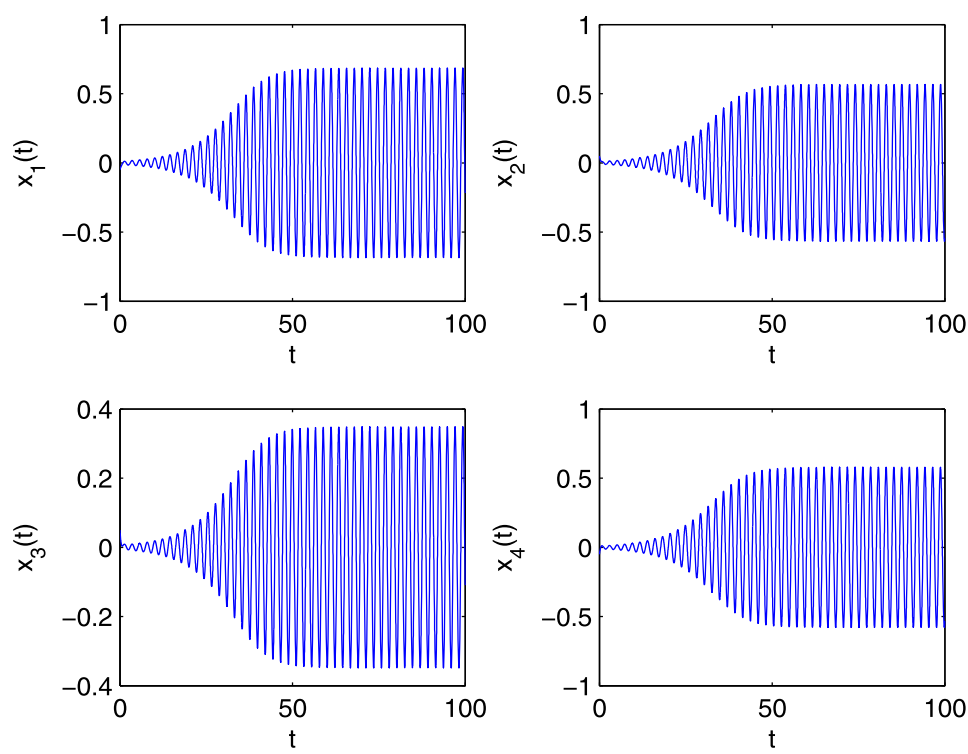

Figure 16 State response of system (5.4) with $\phi=0.96, \tau=0.28>\tau_{0}=0.2427$

Taking the order and initial values as $\phi=0.96$ and $\left(x_{1}(0), x_{2}(0), x_{3}(0), x_{4}(0)\right)=(-0.05$, $0.05,0.05,-0.05)$, respectively, we can have $\omega_{0}=1.8697$, and then $\tau_{0}=0.4486$. Thus, the zero equilibrium point of system (3.2) is global asymptotically stable when $\tau=0.38<\tau_{0}$ (see Figs. 10-11), and when $\tau=0.46>\tau_{0}$, system (5.3) at the zero equilibrium point is unstable (see Figs. 12-13).

If leakage delay is considered in system (5.3), we will give the following example to demonstrate its impact. 

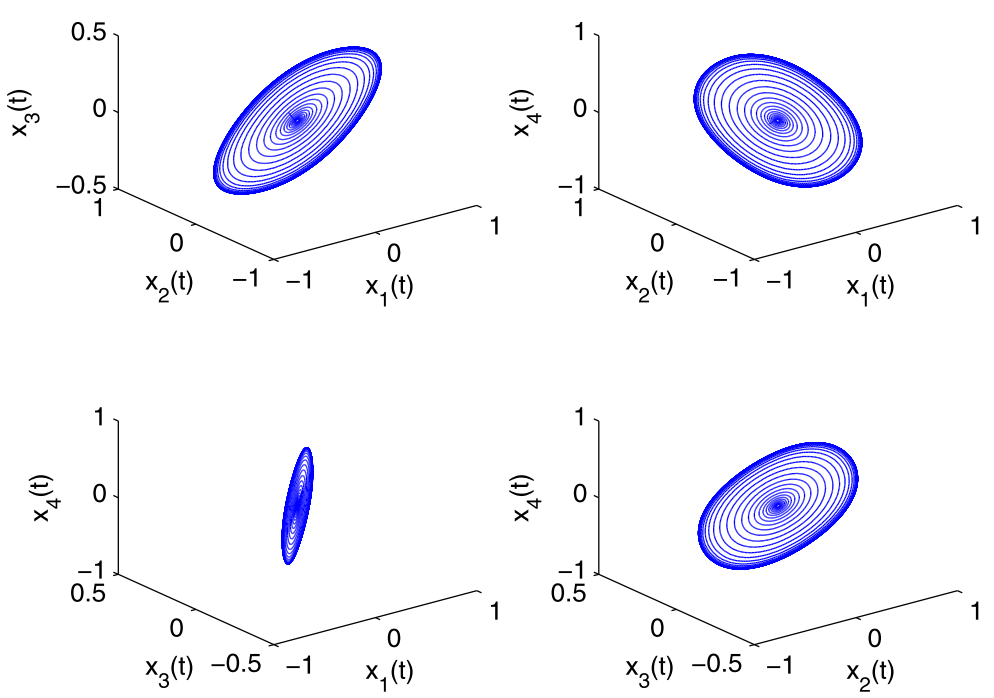

Figure 17 Phase portrait of system (5.4) with $\phi=0.96, \tau=0.28>\tau_{0}=0.2427$

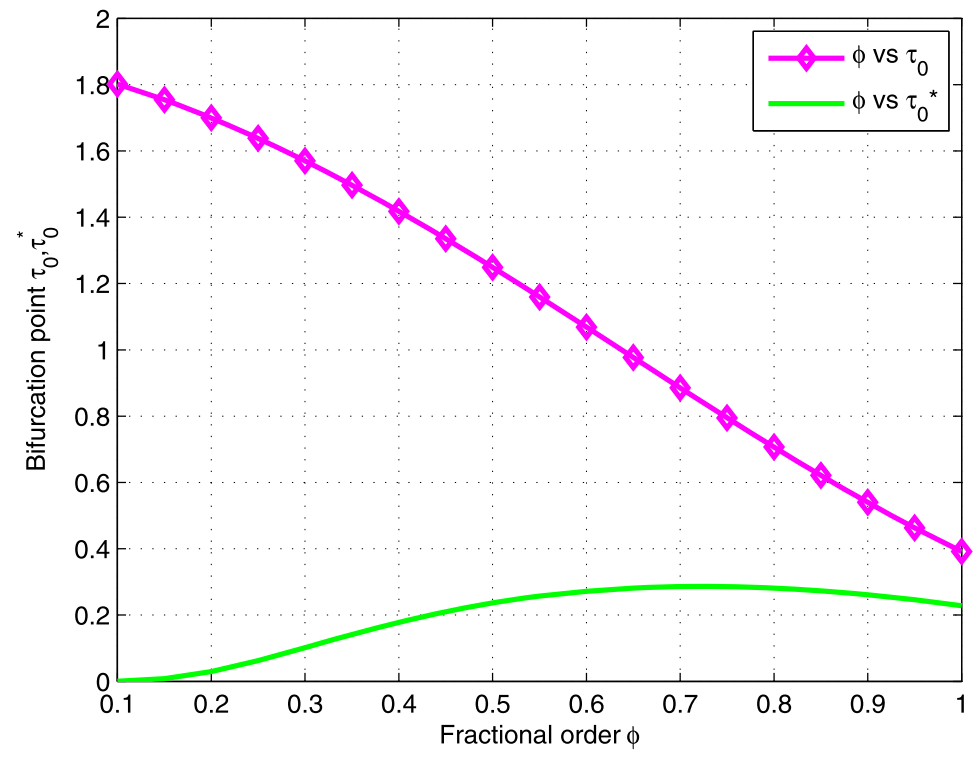

Figure 18 Effects of $\phi$ on $\tau_{0}$ in system (5.3) and $\tau_{0}^{*}$ of system (5.4)

\subsection{Example 4}

Consider the following system with leakage delays:

$$
\left\{\begin{array}{l}
D^{\phi} x_{1}(t)=x_{1}(t-\tau)-0.8 \tanh \left(x_{1}(t)\right)+1.8 \tanh \left(x_{4}(t-\tau)\right)+1.5 \tanh \left(x_{2}(t-\tau)\right), \\
D^{\phi} x_{2}(t)=1.2 x_{2}(t-\tau)-0.8 \tanh \left(x_{2}(t)\right)-1.5 \tanh \left(x_{1}(t-\tau)\right)-1.6 \tanh \left(x_{3}(t-\tau)\right), \\
D^{\phi} x_{3}(t)=0.9 x_{3}(t-\tau)-0.8 \tanh \left(x_{3}(t)\right)+0.5 \tanh \left(x_{2}(t-\tau)\right)+1.2 \tanh \left(x_{4}(t-\tau)\right), \\
D^{\phi} x_{4}(t)=1.5 x_{4}(t-\tau)-0.8 \tanh \left(x_{4}(t)\right)-1.2 \tanh \left(x_{3}(t-\tau)\right)-1.8 \tanh \left(x_{1}(t-\tau)\right) .
\end{array}\right.
$$


The same order and initial values are chosen as those in system (5.3). We now get $\omega_{0}=$ 2.9997 and $\tau_{0}^{*}=0.2477$. Therefore, when $\tau=0.22<\tau_{0}^{*}$, system (5.4) at the zero equilibrium point is global asymptotically stable (see Figs. 14-15); when $\tau=0.28>\tau_{0}^{*}$, system (5.4) at the zero equilibrium point is unstable (see Figs. 16-17). Moreover, if the order $\phi$ varies, the corresponding $\omega_{0}, \tau_{0}^{*}$ can be obtained. It can be seen from Fig. 18 that the onset of Hopf bifurcation of system (5.4) is gradually postponed as the order increases.

\section{Conclusion}

In this paper, the issue of bifurcation for a ring of fractional neural networks with four neurons and time delay in leakage terms has been studied. By utilizing time delay as the bifurcation parameter, some criteria to ensure that existence of the Hopf bifurcation for the fractional four neurons networks were established. The analytic results have shown that both the leakage time delay and communication time delay can change the dynamic behavior quantitatively, for example, greatly changing the stability of equilibrium solution, further leading to Hopf bifurcation and oscillation solutions. Moreover, the impact of the order on the creation of bifurcation was also numerically demonstrated. As a continuation of the previously mentioned series of works, our results may enrich our understanding of the bifurcation for delayed ring fractional neural networks. Finally, simulation examples have been performed to illustrate the main results.

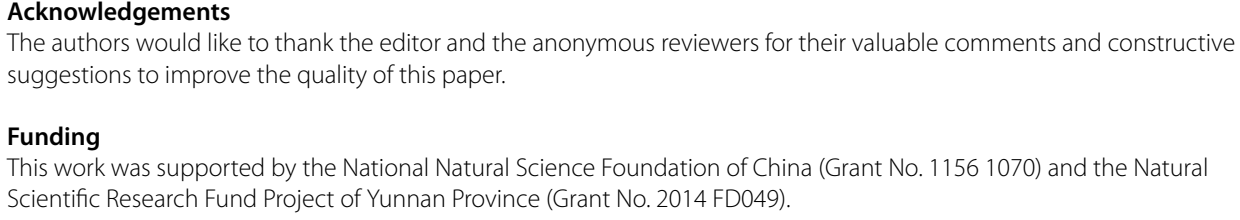

\section{Publisher's Note}

Springer Nature remains neutral with regard to jurisdictional claims in published maps and institutional affiliations.

Received: 21 February 2019 Accepted: 22 April 2019 Published online: 09 May 2019

\section{References}

1. Hopfield, J.: Neural networks and physical systems with emergent collective computational abilities. Proc. Natl. Acad. Sci. USA 79(8), 2554-2558 (1982)

2. Kobayashi, M.: Hyperbolic Hopfield neural networks with directional multistate activation function. Neurocomputing 275, 2217-2226 (2018)

3. Wan, L., Zhou, Q.H., Liu, J.: Delay-dependent attractor analysis of Hopfield neural networks with time-varying delays. Chaos Solitons Fractals 101, 68-72 (2017) 
4. Rech, P.: Chaos and hyperchaos in a Hopfield neural network. Neurocomputing 74(17), 3361-3364 (2011)

5. Coolen, A., Del, P.: Statistical mechanics beyond the Hopfield model: solvable problems in neural network theory. Rev Neurosci. 14(1-2), 181-194 (2003)

6. Zhou, Y., Li, C., Huang, T., Wang, X.: Impulsive stabilization and synchronization of Hopfield-type neural networks with impulse time window. Neural Comput. Appl. 28(4), 775-782 (2017)

7. Chaouki, A.: Oscillation of impulsive neutral delay generalized high-order Hopfield neural networks. Neural Comput. Appl. 29(9), 477-495 (2018)

8. Guo, S., Huang, L.: Hopf bifurcating periodic orbits in a ring of neurons with delays. Physica D 183(1-2), 19-44 (2003)

9. Cao, Y.: Bifurcations in an Internet congestion control system with distributed delay. Appl. Math. Comput. 347, 54-63 (2019)

10. Cao, J., Guerrini, L., Cheng, Z.: Stability and Hopf bifurcation of controlled complex networks model with two delays. Appl. Math. Comput. 343, 21-29 (2019)

11. Smith, K., Wang, L.: Chaos in the discretized analog Hopfield neural network and potential applications to optimization. Protein Sci. 2(2), 1224-1231 (1998)

12. Penn, Y., Segal, M., Moses, E.: Network synchronization in hippocampal neurons. Proc. Natl. Acad. Sci. USA 113(12), $3341-3346(2016)$

13. Pradeepa, C., Cao, Y., Murugesuc, R., Rakkiyappan, R.: An event-triggered synchronization of semi-Markov jump neural networks with time-varying delays based on generalized free-weighting-matrix approach. Math. Comput. Simul. 155, 41-56 (2019)

14. Biggio, M., Storace, M., Mattia, M.: Non-instantaneous synaptic transmission in spiking neuron networks and equivalence with delay distribution. BMC Neurosci. 14(Suppl 1), P267 (2013)

15. Xia, Y., Romanovski, V.: Bifurcation analysis of a population dynamics in a critical state. Bull. Malays. Math. Sci. Soc. 38(2), 499-527 (2015)

16. Rakkiyappan, R., Vinodkumar, A., Rihan, F.: Dynamic analysis for high-order Hopfield neural networks with leakage delay and impulsive effects. Neural Comput. Appl. 22(1), 55-73 (2013)

17. Li, Y., Meng, X., Xiong, L.: Pseudo almost periodic solutions for neutral type high-order Hopfield neural networks with mixed time-varying delays and leakage delays on time scales. Int. J. Mach. Learn. Cybern. 8(6), 1915-1927 (2017)

18. Banu, L., Balasubramaniam, P., Ratnavelu, K.: Robust stability analysis for discrete-time uncertain neural networks with leakage time-varying delay. Neurocomputing 151, 808-816 (2015)

19. Lakshmanan, S., Ju, H., Lee, T.: Stability criteria for BAM neural networks with leakage delays and probabilistic time-varying delays. Appl. Math. Comput. 219(17), 9408-9423 (2013)

20. Popa, C.: Global exponential stability of octonion-valued neural networks with leakage delay and mixed delays. Neural Netw. 105, 277-293 (2018)

21. Gopalsamy, K.: Leakage delays in BAM. J. Math. Anal. Appl. 325(2), 1117-1132 (2007)

22. Zhu, H., Rakkiyappan, R., Li, X.: Delayed state-feedback control for stabilization of neural networks with leakage delay. Neural Netw. 105, 249-255 (2017)

23. Hu, H., Huang, L.: Stability and Hopf bifurcation analysis on a ring of four neurons with delays. Appl. Math. Comput. 213(2), 587-599 (2009)

24. Xu, C., Zhang, Q.: Anti-periodic solutions in a ring of four neurons with multiple delays. Int. J. Comput. Math. 92 1086-1100 (2015)

25. Song, Y., Han, Y., Peng, Y.: Stability and Hopf bifurcation in a unidirectional ring of $n$ neurons with distributed delays. Neurocomputing 121(2), 442-452 (2013)

26. $\mathrm{Ge}, \mathrm{J.}, \mathrm{Xu}, \mathrm{J}$.: Stability and Hopf bifurcation on four-neuron neural networks with inertia and multiple delays. Neurocomputing 287, 34-44 (2018)

27. Deng, W. Li, C., Lu, J.: Stability analysis of linear fractional differential system with multiple time delays. Nonlinear Dyn 48(4), 409-416 (2007)

28. Podlubny, l.: Fractional Differential Equations. Academic Press, New York (1999)

29. Matignon, D.: Stability results for fractional differential equations with applications to control processing. In: IEEE-SMC Pro., Lille, France, vol. 2, pp. 963-968 (1996)

30. Kilbas, A., Srivastava, H., Trujillo, J.: Theory and Application of Fractional Differential Equations. Elsevier, New York (2006)

31. Cao, Y., Li, Y., Ren, W., Chen, Y.: Distributed coordination of networked fractional-order systems. IEEE Trans. Syst. Man Cybern. 40(2), 362-370 (2010)

32. Wang, H., Yu, Y., Wen, G., Zhang, S.: Stability analysis of fractional-order neural networks with time delay. Neural Process. Lett. 42(2), 479-500 (2015)

33. Huang, C., Cao, J., Xiao, M.: Hybrid control on bifurcation for a delayed fractional gene regulatory network. Chaos Solitons Fractals 87, 19-29 (2016)

34. Sun, Q., Xiao, M., Tao, B., Jiang, G., Cao, J., Zhang, F., Huang, C.: Hopf bifurcation analysis in a fractional-order survival red blood cells model and $P D^{\alpha}$ control. Adv. Differ. Equ. 2018(1), 10 (2018)

35. Lundstrom, B., Higgs, M., Spain, W., Fairhall, A.: Fractional differentiation by neocortical pyramidal neurons. Nat. Neurosci. 11(11), 1335-1342 (2008)

36. Xiao, M., Zheng, W., Jiang, G., Cao, J.: Stability and bifurcation of delayed fractional-order dual congestion control algorithms. IEEE Trans. Autom. Control 62, 4819-4826 (2017)

37. Xiao, M., Zheng, W., Jiang, G., Cao, J.: Undamped oscillations generated by Hopf bifurcations in fractional-order recurrent neural networks with Caputo derivative. IEEE Trans. Neural Netw. Learn. Syst. 26(12), 3201-3214 (2015)

38. Huang, C., Meng, Y., Cao, J.: New bifurcation results for fractional BAM neural network with leakage delay. Chaos Solitons Fractals 100, 31-44 (2017)

39. Xiao, M., Jiang, G., Cao, J., Zhang, W.: Local bifurcation analysis of a delayed fractional-order dynamic model of dual congestion control algorithms. IEEE/CAA J. Autom. Sin. 4(2), 361-369 (2017)

40. Bhalekar, S., Varsha, D.: A predictor-corrector scheme for solving nonlinear delay differential equations of fractional order. J. Fract. Calc. Appl. 1, 1-9 (2011) 